African Crop Science Journal by African Crop Science Society is licensed under a Creative Commons Attribution 3.0 Uganda License. Based on a work at www.ajol.info/ and www.bioline.org.br/cs DOI: https://dx.doi.org/10.4314/acsj.v28i2.12

\title{
DEVELOPMENT OF A PRIORITISED CHECKLIST OF CROP WILD RELATIVES FOR CONSERVATION IN MALAWI
}

\author{
N.K. MPONYA ${ }^{1,2}$, Z.L.K. MAGOMBO ${ }^{3}$, L. PUNGULANI ${ }^{1}$, J.M. BREHM ${ }^{2}$ and N. MAXTED ${ }^{2}$ \\ ${ }^{1}$ Malawi Plant Genetic Resources Centre, Chitedze Agricultural Research Station, P. O. Box 158, \\ Lilongwe, Malawi \\ ${ }^{2}$ School of Biosciences, University of Birmingham, B15 2TT, Birmingham, UK. \\ ${ }^{3}$ National Herbarium and Botanic Gardens of Malawi, P. O. Box 528, Zomba, Malawi \\ Corresponding author: noliemponya@yahoo.com
}

(Received 9 October 2019; accepted 26 June 2020)

\begin{abstract}
The national increase in human population in Sub-Saharan Africa (SSA) demands for more food; while increase in the impact of climate change, demands for resilient agricultural production systems, and both call for improved agricultural productivity. Plant breeders will need adaptive traits to improve crop productivity and resilience. Crop wild relatives (CWR) have the potential to offer the much needed diversity for crop improvement, but their diversity is inadequately conserved. Lack of knowledge about their occurrence in Malawi, limits their systematic conservation and utilisation. Developing a CWR national inventory helps to define conservation priorities and actions. The objective of this study was to match checklists of crop genera and national flora, using their taxonomic and genetic relatedness information. This resulted into the first comprehensive annotated checklist of $446 \mathrm{CWR}$ taxa in Malawi, which was prioritised by a set of criteria previously agreed with national stakeholders based on socio-economic importance of the related crop, potential use of the wild relative in crop improvement and threat status. The inventory comprises of 277 CWR taxa, identified as priority for conservation in Malawi; of which $78 \%$ were native. These belong to 56 genera and are related to 54 food, fodder, spices and beverage crops; and include taxa related to crops of regional and global importance. Eighty-seven taxa of highest priority for conservation were further identified, $12.6 \%$ of which have confirmed uses in crop improvement on pests and disease resistance, drought tolerance and yielding ability. The inventory will facilitate effective conservation and availability of these taxa for their use in crop improvement.
\end{abstract}

Key Words: Annotated checklist, national inventory, systematic conservation

\section{RÉSUMÉ}

L'augmentation nationale de la population humaine en Afrique sub-saharienne (ASS) demande plus de nourriture; tandis que l'augmentation de l'impact du changement climatique, la demande de systèmes de production agricole résilients, et tous les deux appellent à une productivité agricole améliorée. Les 
sélectionneurs auront besoin de traits adaptatifs pour améliorer la productivité et la résilience des cultures. Les espèces sauvages apparentées aux cultures (CWR) ont le potentiel d'offrir la diversité nécessaire à l'amélioration des cultures, mais leur diversité est insuffisamment conservée. Le manque de connaissances sur leur présence au Malawi limite leur conservation et leur utilisation systématiques. L'élaboration d'un inventaire national CWR aide à définir les priorités et les actions de conservation. L'objectif de cette étude était de correspondre les listes de contrôle des genres de cultures et de la flore nationale, en utilisant leurs informations de parenté taxonomique et génétique. Cela a abouti à la première liste de contrôle annotée complète de 446 taxons CWR au Malawi, qui a été priorisée par un ensemble de critères préalablement convenus avec les parties prenantes nationales en fonction de l'importance socio-économique de la culture apparentée, de l'utilisation potentielle du parent sauvage

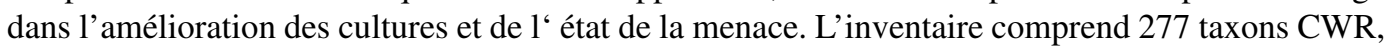
identifiés comme prioritaires pour la conservation au Malawi; dont $78 \%$ étaient indigènes. Ils appartiennent à 56 genres et sont liés à 54 cultures vivrières, fourragères, d'épices et de boissons; et inclure les taxons liés aux cultures d'importance régionale et mondiale. Quatre vingt sept taxons prioritaires pour la conservation ont été identifiés, dont $12,6 \%$ ont confirmé des utilisations dans l'amélioration des cultures contre les ravageurs et la résistance aux maladies, la tolérance à la sécheresse et la capacité de rendement. L'inventaire facilitera la conservation efficace et la disponibilité de ces taxons pour leur utilisation dans l'amélioration des cultures.

Mots Clés: Liste de contrôle annotée, inventaire national, conservation systématique

\section{INTRODUCTION}

Crop wild relatives (CWR) have potential for contributing to improved global food and economic security in that they are donors of adaptive genes for crop improvement (Harlan and de Wet, 1971; Hajjar and Hodgkin, 2007). Maxted et al. (2006) defined CWR as wild plant species, genetically close to cultivated plants. The use of CWR in improving crop adaptation to abiotic and biotic stresses dates back to 1800's (Ramdoyal and Badaloo, 2002). Evidence of gene transfer from CWR to cultivated plants was reported in a number of studies (Hajjar and Hodgkin, 2007; Maxted and Kell, 2009; Ishimaru et al.,2010), and therefore, the need to manage the diversity in CWR and make it available and accessible to plant breeders at all levels is inevitable

The need to conserve CWR is also recognised in global instruments such as Global Plan of Action of the Food and Agriculture Organisation of the United Nations (FAO, 2012); the Sustainable Development Goals 2, sub-item 2.5 and 15 sub items 15.4, 15.5 and 15.6 (UN, 2015). It is also echoed in
Aichi targets on Biodiversity Strategic goal C, Targets 11, 12 and 13 (https://www.cbd.int/ sp/targets/), the Convention on Biological Diversity (CBD), Global Strategy on Plant Conservation (GSPC) (CBD, 2000), the International Treaty on Plant Genetic Resources for Food and Agriculture (ITPGRFA) (FAO, 2009) and the joint notification of the CBD/ITPGRFA/CGRFA/ Bioversity (CBD, 2019) that countries like Malawi area party to.

The national gene bank of Malawi manages the gene pool diversity of local crops, in an effort to improve utilisation of genetic diversity in the national breeding programmes. But due to limited resources, conservation thus far, has only covered landraces, excluding CWR diversity. The FAO (1996) State of the Country report on plant genetic resources, indicates the occurrence of some CWR in Malawi, but to use these in crop improvement programme requires effective conservation (Dempewolf et al., 2014). The objective of this study was to develop a national inventory of CWR based on their native status, national and global distribution (rarity and/or endemicity), threat 
status, potential use in crop improvement, and importance of related crop to facilitate their conservation and use.

\section{MATERIALS AND METHODS}

Crop wild relatives general checklist. A floristic approach was used in the development of Malawi's CWR checklist. The procedure followed those outlined in the Interactive Toolkit for CWR Conservation Planning (Brehm et al., 2017). First, a crop genera checklist was compiled, with information from Flora of Malawi, using cultivated plant families (Hyde et al., 2018); and useful plants in Malawi (edible and cultivated) (Williamson, 2005). Crops of global economic importance from Annex 1 of the ITPGRFA (FAO, 2009), crops listed in FAOSTAT (FAOSTAT, 2016); crops from national agricultural production estimates and a crop checklist from the Malawi Plant Genetic Resources Centre (MPGRC) accession database were also used. The crop genera checklist included crops cultivated and those not cultivated in Malawi,but were of regional and global importance and have wild relatives occurring in Malawi. The main reason for including crops not cultivated in Malawi was to capture CWR diversity that under pins the Southern Africa Development Community (SADC) and global food security (FAO, 2009; Allen et al., 2019).

Second, a national flora checklist was compiled with data from global and national databases, which included The Royal Botanic Gardens-Kew (2017), Global Biodiversity Information Facility (GBIF, 2017), the Flora of Malawi (Hyde et al., 2018), National Herbarium and Botanic Gardens of Malawi, Useful plants of Malawi (Williamson, 2005) and MPGRC as these maintain collections of wild species of Malawi. For herbarium specimens, the process involved image capture, digitisation and taxonomic name check from the Plant List (The Plant List, 2013).
Finally, the national flora checklist was matched against the crop genera checklist to produce a national complete CWR checklist (Fig.1). The checklist was annotated with crop commodity groups' information, e.g. food, fodder, beverage, oil and food, fiber crops, etc., based on the Department of Agricultural Research Services (DARS) System, with reference to FAO (n.d.) crop commodity groups' classification. Information about related crops, gene pool and taxon group concepts were sourced from the USDA, Agricultural Research Service, National Plant Germplasm System (2018) and the Harlan de Wet inventory (Vincent et al., 2013) guided by Harlan and de Wet (1971) and Maxted et al. (2006), respectively. National and global distributions were sourced from Flora of Malawi (Hyde et al., 2018) and GRIN Taxonomy (USDA, Agricultural Research Service, National Plant Germplasm System, 2018) and Red List threat status information sourced from plants red list data sources (Raimondo et al., 2009; IUCN, 2018).

The Plant List (2013), the USDA, Agricultural Research Service, National Plant Germplasm System (2018) and Wiersema and León (2016) were instrumental in sorting out species nomenclature and synonyms. The checklist was then compared with the inventory of priority CWR of the SADC region (Allen et al., 2017; Allen et al., 2019) developed through the SADC Crop Wild Relatives project (http://www.cropwild relatives.org/), to ensure that taxa of SADC regional importance were captured.

\section{Prioritisation of the CWR checklist to} develop a national inventory of CWR. The prioritisation process involved relevant national stakeholders, and was carried out in two steps (Fig.1); namely (i) defining crops regarded as of high priority for Malawi and their wild relatives; and (ii) prioritisation of the wild relatives of crops of low priority to Malawi (Fig. 2). The process is as described below. 


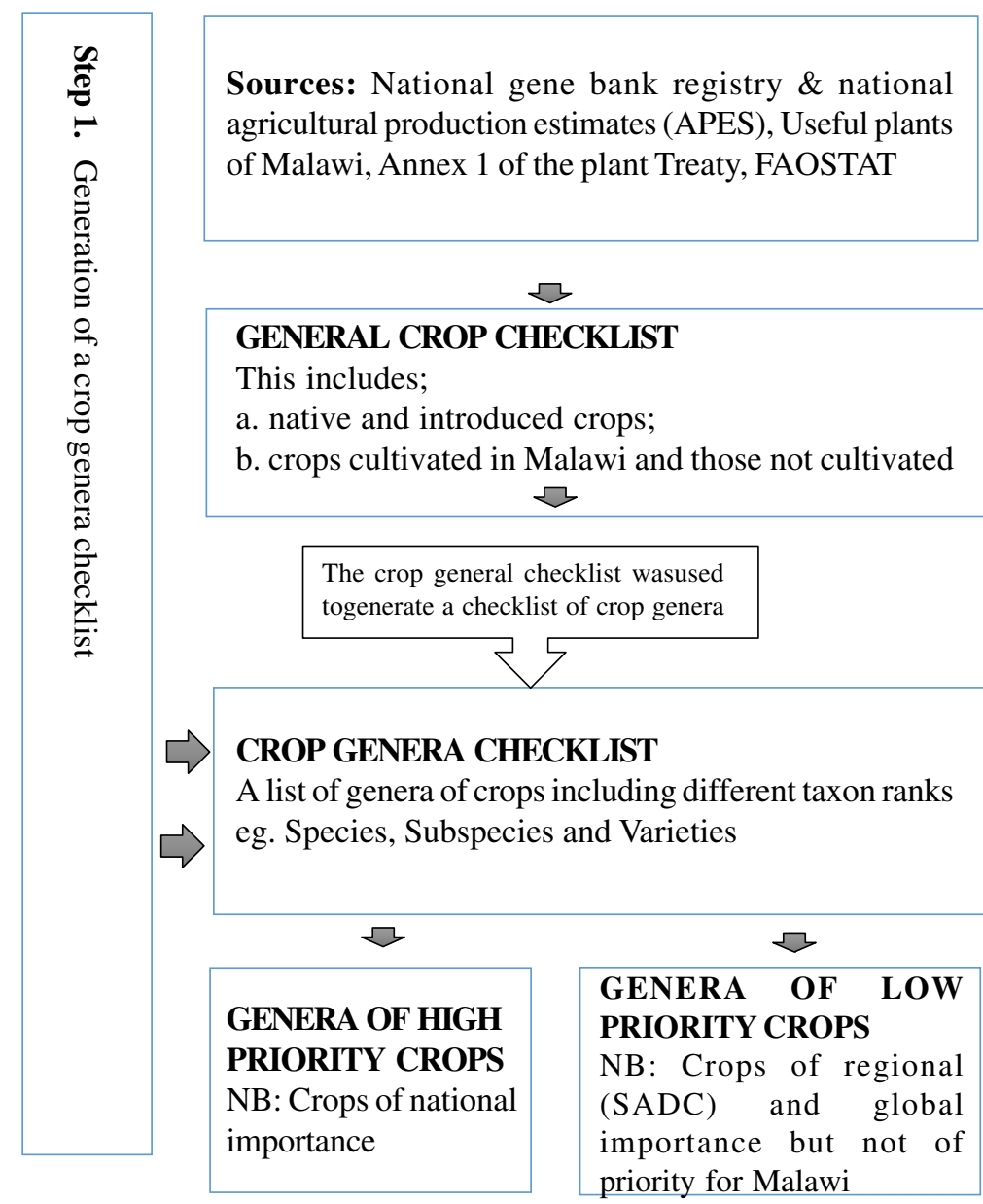

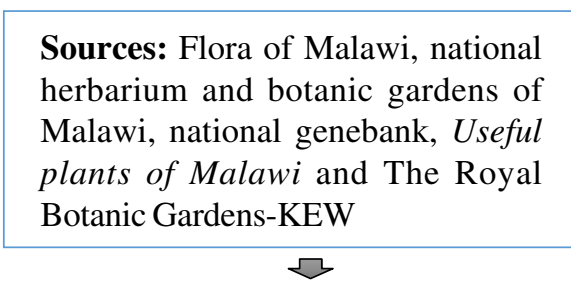

ACHECKLISTOFFLORAOFMALAWI

\section{ए \\ GENERAL CWR CHECKLIST OF MALAWI}

This includes;

1. Checklist of CWR from high priority crops to Malawi.

2. Checklist of CWR from low priority crops to Malawi but of regional and global importance.

NB: This was annotated.

Figure 1. Processes in generation of Crop Wild Relatives (CWR) checklist for Malawi. 
Step 2. Prioritization of CWR of low priority crops of Malawi Criteria: Related crop value, species distribution (national and global), taxon native status, threat status and potential use in crop improvement. NB: (National stakeholders defined some criteria)

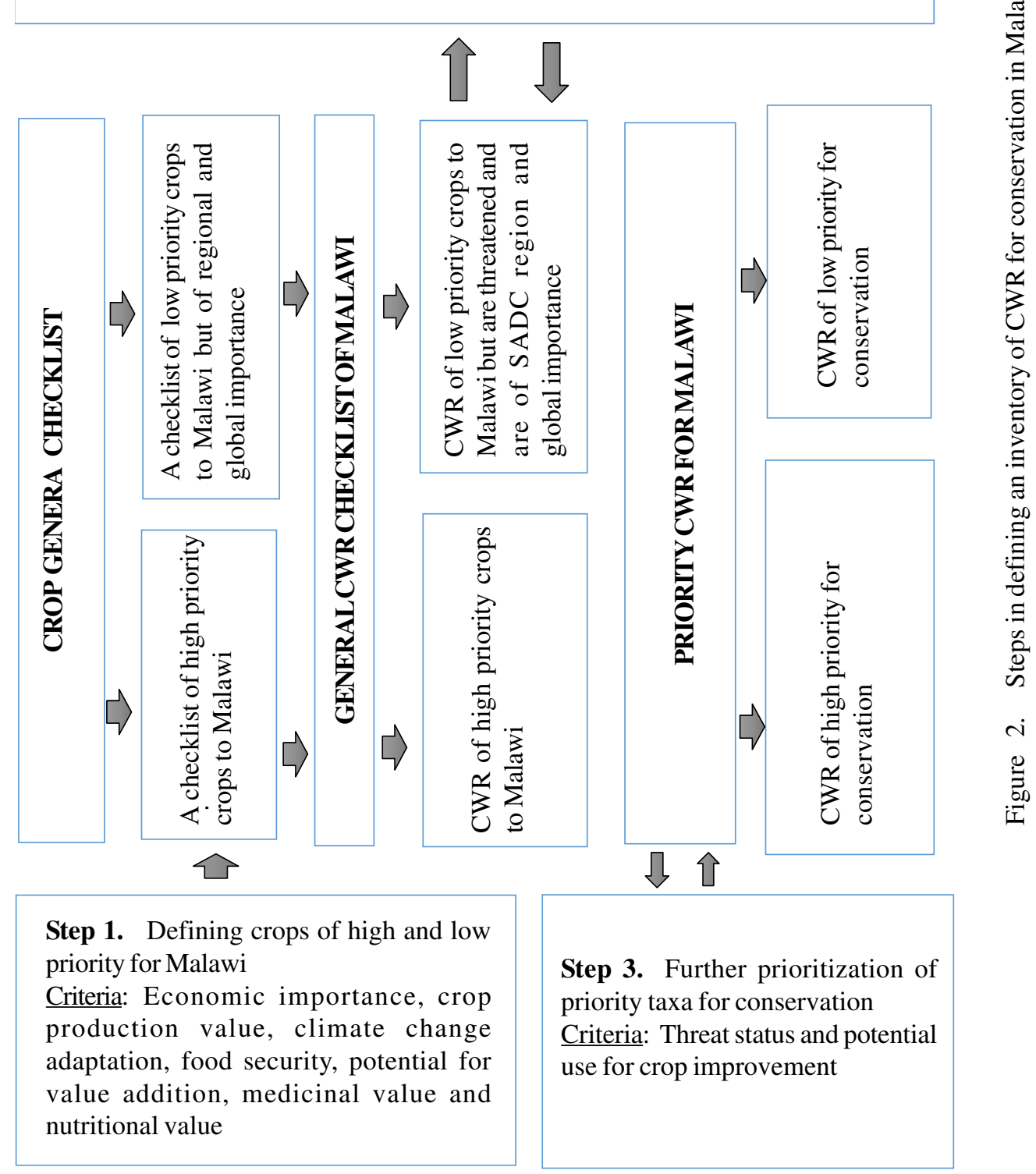


Defining wild crops relatives of priority to Malawi. National stakeholders were involved in identifying crops that were priority to food security in Malawi. This was during a National Stakeholder Consultative Workshop held on the $19^{\text {th }}$ October 2017 at Silver Sands Resort in Salima, Malawi. Experts included those from the fields of plant breeding, crop production (field and horticultural crops), pasture agronomy, taxonomy, statistics, natural resources conservation, and those responsible for national plant genetic resources conservation. Stakeholders were involved in order to bring in relevance of the checklist to the users and to encourage ownership and use of the priority checklist in national conservation and utilisation of CWR. The selection of the crops regarded as priority to Malawi were based on the following:

(i) Crop economic importance. Role of the crop as foreign exchange earner and ability to boost local economy based on national economic analysis reports (FAO, 2019);

(ii) Food security. Main food and fodder crops with multiple uses (used raw, processed and its by-product) and that are utilised in the country across seasons measured by production quantity and foreign exchange value (FAO, 2019);

(iii) Climate change adaptation. Crops known to be adaptable to extreme weather conditions; in Malawi e.g. sorghum is associated with drought tolerance;

(iv) Nutritional value. Crops mainly regarded as of high nutritional content and are readily available to the majority of the population in Malawi;

(v) Medicinal value. Crops with benefits to human health; and (vi) Potential for value addition. Crops with potential for commercialisation.

Crops were matched against each criterion listed above, and those that qualified for one or more of the six criteria were regarded as of high importance to Malawi; hence of high priority and therefore their CWR were also regarded as priority for conservation in Malawi.

\section{Prioritisation of the wild relatives of crops of low priority to Malawi}

Prioritisation of the wild relatives of low priority crops to Malawi was carried out in order to capture CWR taxa that were threatened, with the aim of rescuing them and taxa of regional (SADC) and global importance. Prioritisation criteria used were a combination from those suggested by Hunter and Heywood (2011), as well as those used to prioritise Jordan vascular plants species (Magos Brehm et al., 2016). Five criteria were selected for the prioritisation. Additional information such as taxon nativeness, threat status, geographic distribution and gene pool and taxonomic groups in relation to the CWR prioritisation criteria was gathered. Taxa that qualified for one or more criteria below were selected as priority for conservation.

Taxon native status. Taxa known to be native to Malawi or introduced to the country and adapted to local conditions, but not invasive, the native species were prioritised.

Taxon national distribution. This was based on the taxon range distribution within the country based on number of regions of occurrence. Taxa with wide range distribution had a chance of surviving than those with restricted range distribution; and these may be rare or endemic hence, were given higher priority for conservation 
Taxon global distribution. This refers to worldwide distribution of the taxon. The likelihood of losing taxa with restricted geographic distribution due to localised threats and climate change impacts is high compared to those with a wide range distribution; and hence the former must receive more conservation attention than the latter. This was categorised as: (i) endemic to Malawi; (ii) occurring in Malawi plus two countries in the SADC region; (iii) endemic to the SADC region, (iv) occurring in all tropical African countries and outside Africa. For this, priority was given to taxa endemic to Malawi.

Potential use of taxon in crop improvement. Taxonomic and genetic relatedness of taxon to the crop based on taxon and gene pool group concepts determines how easily these wild relatives can be used for crop improvement (Harlan and de Wet, 1971; Maxted et al., 2006). GRIN taxonomy (USDA, Agricultural Research Service, National Plant Germplasm System., 2018), the Harlan and de Wet CWR inventory (Vincent et al., 2013) and literature (Plaza et al., 2014) were the sources of the required information. For taxa whose gene pools were not explicitly documented, the taxon group concept proposed by Maxted et al. (2006) was used to assign species taxonomic groups based on the classification information about the taxa. This was done by matching CWR taxa with the genus, subgenus, and species and/or series of its cultivated taxa based on a general definition of a CWR. Species that fall in TG1b, TG2 and TG3 and those in GP1b and GP2, regardless of their native and the assigned global or national threat status were considered of high priority for conservation as they have highest potential use in crop improvement (Harlan and de Wet, 1971; Maxted et al., 2006)

Taxon threat status. Level of threat of the wild relative based on the Global IUCN Red Listing found on:http://www.iucnredlist.org, and South African plants (Raimondo et al.,
2009). We used South Africa red listing results because no recent threat assessments on vascular plants have been done in Malawi. Moreover, Malawi's 2002 species red listing (Dombo et al., 2012), included only one taxon for CWR. South Africa was an alternative due to its record of high diversity of flora in the SADC region, and its assessments included substantial number of species. Therefore, to have an overview of species threat levels within the SADC region and at global level, red listing results by IUCN and South Africa were used as proxy indicator for the threat status of Malawi's CWR species. Species that are Critically Endangered (CR), Endangered (EN), Vulnerable (VU) and Near threatened (NT) were of high priority for conservation regardless of their taxonomic, gene pool concept and national priority category.

\section{RESULTS}

The general CWR checklist. The crop genera checklist had 113 crops (91 cultivated and 22 not cultivated in Malawi) (Table 1). The flora of Malawi checklist had 1,173 taxa; and after matching against the crop genera checklist, 446 taxa were identified as CWR related to 61 crops, belonging to 68 genera within 22 families. Out of the 446 CWR, 74.7\% of the taxa were native to Malawi. The largest numbers of taxa were recorded in the families of Poaceae (133), Leguminosae (83), Lamiaceae (42), Convolvulaceae (34) and Solanaceae (33). About $60 \%$ of the CWR in the checklist were related to food crops, $26 \%$ to fodder crops, and $8 \%$ to crops in the category of spices; while $6 \%$ belonged to confectionery, fiber and oil seed crops.

Eight and half percent of 446 taxa had been assessed in the IUCN Red List of Threatened Species and the South African Plants Red Listing (Raimondo et al., 2009) included 34.8 with $4.3 \%$ of the 446 species, being assessed at both global level and in South Africa. Together, these assessments reported eight 
TABLE 1. Crops and crop genera used to generate a general checklist of crop wild relatives occurring in Malawi with their cultivation status and priority levels based on the prioritisation criteria

\begin{tabular}{|c|c|c|c|}
\hline Crop & Genus & $\begin{array}{l}\text { Cultivation status } \\
\text { in Malawi }\end{array}$ & Priority level \\
\hline Acorn Squash & Cucurbita & $\mathrm{C}$ & LP \\
\hline Adzuki Bean & Vigna & $\mathrm{C}$ & LP \\
\hline Air yam & Dioscorea & $\mathrm{C}$ & LP \\
\hline Almond & Prunus & $\mathrm{C}$ & LP \\
\hline Amaranth & Amaranthus & $\mathrm{C} / \mathrm{W}$ & $\mathrm{HP}$ \\
\hline Apple & Malus & $\mathrm{C}$ & $\mathrm{LP}$ \\
\hline Asparagus & Asparagus & $\mathrm{C}$ & $\mathrm{HP}$ \\
\hline Bambara Groundnut & Vigna & $\mathrm{C}$ & HP \\
\hline Banana & Musa & $\mathrm{C}$ & HP \\
\hline Barley & Hordeum & $\mathrm{NC}$ & $\mathrm{HP}$ \\
\hline Beet & Beta & $\mathrm{C}$ & LP \\
\hline Black Mustard & Brassica & $\mathrm{C}$ & LP \\
\hline Black pepper & Piper/Peperomia & $\mathrm{C}$ & LP \\
\hline Blue berries & Vaccinium & $\mathrm{C}$ & LP \\
\hline Bread fruit & Treculia & $\mathrm{C}$ & LP \\
\hline Breadfruit/Jackfruit & Artocarpus & $\mathrm{C} / \mathrm{W}$ & LP \\
\hline Cabbage & Brassica & $\mathrm{C}$ & LP \\
\hline Cacao & Theobroma & $\mathrm{NC}$ & LP \\
\hline Cardamom & Aframomum & $\mathrm{C}$ & LP \\
\hline Carrot & Daucus & $\mathrm{C}$ & LP \\
\hline Cashew & Anacardium & $\mathrm{C}$ & HP \\
\hline Cassava & Manihot & $\mathrm{C}$ & $\mathrm{HP}$ \\
\hline Castor oil & Ricimus & $\mathrm{W}$ & LP \\
\hline Cat's whiskers & Cleome & $\mathrm{C} / \mathrm{W}$ & LP \\
\hline Centro & Centrosema & $\mathrm{C}$ & LP \\
\hline Cherry & Prunus & $\mathrm{C}$ & LP \\
\hline Chickpea & Cicer & $\mathrm{C}$ & LP \\
\hline Cinnamon & Cinnamomum & $\mathrm{C}$ & HP \\
\hline Coco yam/taro & Colocasia & $\mathrm{C}$ & LP \\
\hline Cocoyam & Xanthosoma & $\mathrm{C}$ & $\mathrm{LP}$ \\
\hline Coffee & Coffea & $\mathrm{C}$ & HP \\
\hline Beans (Common, limabean) & Phaseolus & $\mathrm{C}$ & $\mathrm{HP}$ \\
\hline Cotton & Gossypium & $\mathrm{C}$ & HP \\
\hline Cowpea & Vigna & $\mathrm{C}$ & HP \\
\hline Kaki/Persimmon & Diospyros & $\mathrm{NC}$ & LP \\
\hline Cucumber & Cucumis & $\mathrm{C}$ & LP \\
\hline Date palm & Phoenix & $\mathrm{C}$ & LP \\
\hline Desmodium & Desmodium & $\mathrm{C} / \mathrm{W}$ & LP \\
\hline Eggplant & Solanum & $\mathrm{C}$ & LP \\
\hline Faba Bean & Vicia & $\mathrm{C}$ & $\mathrm{HP}$ \\
\hline Finger Millet & Eleusine & $\mathrm{C}$ & HP \\
\hline Fish bean & Tephrosia & $\mathrm{C} / \mathrm{W}$ & LP \\
\hline Garden peas & Pisum & $\mathrm{C}$ & LP \\
\hline Garlic & Allium & $\mathrm{C}$ & LP \\
\hline Ginger & Zingiber & $\mathrm{C}$ & HP \\
\hline Gourds & Lagenaria & $\mathrm{C}$ & LP \\
\hline
\end{tabular}


TABLE 1. Contd.

\begin{tabular}{|c|c|c|c|}
\hline Crop & Genus & $\begin{array}{l}\text { Cultivation status } \\
\text { in Malawi }\end{array}$ & Priority level \\
\hline Grape & Vitis & $\mathrm{C}$ & LP \\
\hline Grapefruit & Citrus & $\mathrm{C}$ & LP \\
\hline Green grams & Vigna & $\mathrm{C}$ & LP \\
\hline Groundnut & Arachis & $\mathrm{C}$ & $\mathrm{HP}$ \\
\hline Hyacinth beans & Lablab & $\mathrm{C}$ & LP \\
\hline Leek & Allium & $\mathrm{C}$ & LP \\
\hline Lemon & Citrus & $\mathrm{C}$ & LP \\
\hline Lentil & Lens & $\mathrm{C}$ & LP \\
\hline Lettuce & Lactuca. & $\mathrm{C}$ & LP \\
\hline Livingstone potato & Plectranthus & $\mathrm{C} / \mathrm{W}$ & $\mathrm{LP}$ \\
\hline Macadamia & Macadamia & $\mathrm{C}$ & HP \\
\hline Macrotyloma & Macrotyloma & $\mathrm{C} / \mathrm{W}$ & LP \\
\hline Maize & $\mathrm{Zea}$ & $\mathrm{C}$ & HP \\
\hline Mango & Mangifera & $\mathrm{C}$ & $\mathrm{LP}$ \\
\hline Melon & Cucumis & $\mathrm{C}$ & LP \\
\hline Millet (Panicum) & Panicum & $\mathrm{C}$ & LP \\
\hline Millet (Setaria) & Setaria & $\mathrm{C}$ & LP \\
\hline Moringa (Drumstick tree) & Moringa & $\mathrm{C}$ & HP \\
\hline Mustard & Brassica & $\mathrm{C}$ & $\mathrm{HP}$ \\
\hline Oat & Avena & $\mathrm{NC}$ & LP \\
\hline Okra & Hibiscus & $\mathrm{C}$ & LP \\
\hline Olive & Olea & $\mathrm{NC}$ & LP \\
\hline Onion & Allium & $\mathrm{C}$ & LP \\
\hline Orange & Citrus & $\mathrm{C}$ & $\mathrm{LP}$ \\
\hline Papaya & Carica & $\mathrm{C}$ & LP \\
\hline Peach & Prunus & $\mathrm{C}$ & LP \\
\hline Pear & Pyrus & $\mathrm{C}$ & $\mathrm{LP}$ \\
\hline Pearl Millet & Pennisetum & $\mathrm{C}$ & HP \\
\hline Pepper & Capsicum & $\mathrm{C}$ & LP \\
\hline Pigeon Pea & Cajanus & $\mathrm{C}$ & HP \\
\hline Pineapple & Ananas & $\mathrm{C}$ & $\mathrm{LP}$ \\
\hline Plum & Prunus & $\mathrm{C}$ & LP \\
\hline Potato & Solanum & $\mathrm{C}$ & HP \\
\hline Pumpkin & Cucurbita & $\mathrm{C}$ & LP \\
\hline Purple bush bean & Macroptilium & $\mathrm{C} / \mathrm{W}$ & LP \\
\hline Quinoa & Chenopodium & $\mathrm{C}$ & LP \\
\hline Rape & Brassica & $\mathrm{C}$ & HP \\
\hline Raspberry & Rubus & $\mathrm{C}$ & LP \\
\hline Rhodes grass & Chloris & $\mathrm{C}$ & $\mathrm{HP}$ \\
\hline Rice & Oryza & $\mathrm{C}$ & HP \\
\hline Rye & Secale & $\mathrm{NC}$ & LP \\
\hline Sesame & Sesamum & $\mathrm{C}$ & HP \\
\hline Sorghum & Sorghum & $\mathrm{C}$ & HP \\
\hline Soybean & Glycine & $\mathrm{C}$ & $\mathrm{HP}$ \\
\hline Spinach & Spinacia & $\mathrm{C}$ & LP \\
\hline Strawberry & Fragaria & $\mathrm{C}$ & LP \\
\hline Sugarcane & Saccharum & $\mathrm{C}$ & HP \\
\hline
\end{tabular}


TABLE 1. Contd.

\begin{tabular}{|c|c|c|c|}
\hline Crop & Genus & $\begin{array}{l}\text { Cultivation status } \\
\text { in Malawi }\end{array}$ & Priority level \\
\hline Sunflower & Helianthus & $\mathrm{C}$ & LP \\
\hline Sweet potato & Ipomoea & $\mathrm{C}$ & $\mathrm{HP}$ \\
\hline Tea & Camellia & $\mathrm{C}$ & HP \\
\hline Teff & Eragrostis & $\mathrm{C}$ & LP \\
\hline Tobacco & Nicotiana & $\mathrm{C}$ & $\mathrm{HP}$ \\
\hline Tomato & Lycopersicon & $\mathrm{C}$ & $\mathrm{LP}$ \\
\hline Turnip & Brassica & $\mathrm{C}$ & LP \\
\hline Urd Bean/Mung bean & Vigna & $\mathrm{C}$ & LP \\
\hline Velvet beans & Mucuna & $\mathrm{C} / \mathrm{W}$ & LP \\
\hline Vetch & Vicia & $\mathrm{C}$ & LP \\
\hline Water melon & Citrullus & $\mathrm{C}$ & LP \\
\hline Water Yam & Dioscorea & $\mathrm{C}$ & LP \\
\hline Wheat & Triticum & $\mathrm{C}$ & LP \\
\hline White Guinea Yam & Dioscorea & $\mathrm{C}$ & LP \\
\hline Yam bean & Sphenostylis & $\mathrm{NC}$ & LP \\
\hline Yellow Yam & Dioscorea & $\mathrm{C}$ & LP \\
\hline Milkvetch & Astragalus & $\mathrm{NC}$ & Annex 1 IT (LP) \\
\hline Jack bean & Canavalia & $\mathrm{NC}$ & Annex 1 IT (LP) \\
\hline Scorpion vetch & Coronilla & $\mathrm{NC}$ & Annex 1 IT (LP) \\
\hline Alpine sweetvetch & Hedysarum & $\mathrm{NC}$ & Annex 1 IT (LP) \\
\hline Grasspea & Lathyrus & $\mathrm{NC}$ & Annex 1 IT (LP) \\
\hline Lespedeza (all varieties) & Lespedeza & $\mathrm{NC}$ & Annex 1 IT (LP) \\
\hline Trefoil & Lotus & $\mathrm{NC}$ & Annex 1 IT (LP) \\
\hline Lupin & Lupinus & $\mathrm{NC}$ & Annex 1 IT (LP) \\
\hline Alfalfa & Medicago & $\mathrm{NC}$ & Annex 1 IT (LP) \\
\hline Melilot, & Melilotus & $\mathrm{NC}$ & Annex 1 IT (LP) \\
\hline Common sainfoin & Onobrychis & $\mathrm{NC}$ & Annex 1 IT (LP) \\
\hline Bird's-foot & Ornithopus & $\mathrm{NC}$ & Annex 1 IT (LP) \\
\hline African mesquite, iron tree & Prosopis & $\mathrm{NC}$ & Annex 1 IT (LP) \\
\hline Puero, Tropical Kudzu & Pueraria & $\mathrm{NC}$ & Annex 1 IT (LP) \\
\hline Clovers & Trifolium & $\mathrm{NC}$ & Annex 1 IT (LP) \\
\hline Broomsedge & Andropogon & $\mathrm{NC}$ & Annex 1 IT (LP) \\
\hline Crested wheatgrass & Agropyron & $\mathrm{NC}$ & Annex 1 IT (LP) \\
\hline Redtop & Agrostis & $\mathrm{NC}$ & Annex 1 IT (LP) \\
\hline Meadow foxtail & Alopecurus & $\mathrm{NC}$ & Annex 1 IT (LP) \\
\hline False oat-grass & Arrhenatherum & $\mathrm{NC}$ & Annex 1 IT (LP) \\
\hline Grass, Orchard & Dactylis & $\mathrm{NC}$ & Annex 1 IT (LP) \\
\hline Blue fescue & Festuca & $\mathrm{NC}$ & Annex 1 IT (LP) \\
\hline
\end{tabular}

IT are crops of global importance according to the International Treaty on Plant Genetic Resources for Food and Agriculture. $\mathrm{LP}=$ Low priority, $\mathrm{HP}=$ High priority, $\mathrm{C}=$ Cultivated, $\mathrm{NC}=$ Not cultivated, $\mathrm{C} / \mathrm{W}$ $=$ Cultivated but also occur in the wild 
taxa being threatened and one taxa was threatened at both levels.

Prioritised crop wild relatives. Out of 113 crops used to generate crop genera, 33 crops were identified as of high priority based on their role in food (including feed) and nutrition security, climate change adaptation and their economic importance and potential for value addition. However, only 24 crops had CWR occurring in Malawi, and these had $158 \mathrm{CWR}$ taxa. Forty one CWR taxa from this group had potential use for crop improvement.

For the 80 low priority crops, only 37 crops had CWR occurring in Malawi. In total, these 37 low priority crops registered occurrence of 288 CWR taxa, of which after prioritisation, 119 taxa were identified as priority for conservation based on the criteria described above; and were related to 30 low priority crops. From this category, thirty-two CWR taxa had potential use in crop improvement, four taxa were endemic to Southern Region of Malawi, one taxa was threatened at global level, and the rest were not assessed but were selected based on their national distribution status.

The prioritised checklist. The national inventory had 277 priority CWR taxa (from both high and low priority crops), and were related to 54 crops from 56 genera across 19 plant families. Most of them were in the families of Leguminosae (79), Poaceae (74), Convolvulaceae (34) and Solanaceae (33); while the rest of the families had less than 20 taxa. About $78 \%$ of the taxa in the national inventory were related to crops that were rated as of high value in terms of food, feed and nutritional security, economic importance and potential for value addition and adaptation to climate change. Examples of such crops included coffee, cotton, cowpeas, rice, sorghum, sugarcane, asparagus, black pepper, sweet potato and cassava (Tables 2 and 3 ).

A total of 164 CWR taxa in the inventory were related to crops of global importance
(Vincent, et al., 2013), 34 taxa were also included as priority in the SADC region (Allen et al., 2017; 2019), and 21 CWR taxa were priority in Malawi, the SADC region and at global level (Table 2).

Out of the 277 taxa, $78 \%$ were native, $5.8 \%$ were introduced to Malawi, and the status of $45(16.3 \%)$ taxa was not specified (Table 2). Although results reveal that several priority taxa had a restricted range distribution within Malawi, $87 \%$ of 277 taxa occurred in more than one country.Within Malawi, Southern region reported the highest diversity of priority taxa (48) that did not occur in other regions; followed by Northern (34) and Central region (12). About $25.6 \%$ of the taxa occurred across the country, and the remainder occurred in one or two regions. It was also noted that Coffea mufindiensis Bridson subsp. pawekiana (Bridson) and $C$. arabica L. wild types were endemic to Southern region, and $C$. mufindiensis Hutch ex Bridson subsp. lundaziensis and Setaria grandis Stapf were near endemic and only found in the Northern region. Other endemic species included Eragrostis fastigiata Cope., E. sylviae Cope. and Plectranthus mandalensis Baker only known from Southern region of Malawi.

Prioritisation of CWR taxa by threat status and the potential use of the wild relative for crop improvement revealed that 34 taxa were assessed for threat status at global level. Of these, 29 taxa were Least Concern (LC), one taxon was assessed as Data Deficient (DD) [Vigna hosei (Craib) Backer], and four species were threatened and these included the wild populations of Coffea arabica L. and C.salvatrix Swynnerton \& Phillipson assessed as Endangered (EN), Prunus africana (Hook.f.) Kalkman, and C.ligustroides S. Moore as Vulnerable (VU).

South African Red List assessments covered $106 \mathrm{CWR}$ taxa of the national inventory; three species were reported threatened and these included Siphonochilus aethiopicus (Schweinf.) B.L.Burtt assessed as Critically Endangered (CR), Prunus africana 
TABLE 2. Crop wild relatives priority for conservation in Malawi and their native status (no=wild relative not priority for the SADC region and is not related to crop of global importance; yes=wild relative of priority to SADC region and is related to crop of global importance)

\begin{tabular}{|c|c|c|c|c|}
\hline Crop & Priority crop wild relatives for Malawi & $\begin{array}{l}\text { Included in } \\
\text { the SADC } \\
\text { inventory } \\
\text { (Yes/No) }\end{array}$ & $\begin{array}{l}\text { Related to } \\
\text { crop of global } \\
\text { importance } \\
\text { (Yes/No) }\end{array}$ & Status \\
\hline \multirow[t]{3}{*}{ Amaranth } & Amaranthus dubius Mart. ex Thell.* & $\mathrm{n}$ & $\mathrm{n}$ & native \\
\hline & A. graecizans L. subsp. Silvestris (Vill.) Brenan* & $\mathrm{n}$ & $\mathrm{n}$ & native \\
\hline & A. hybridus $\mathrm{L}$ & $\mathrm{n}$ & $\mathrm{n}$ & native \\
\hline \multirow[t]{14}{*}{ Asparagus } & Asparagus laricinus Burch. & $\mathrm{n}$ & $\mathrm{y}$ & native \\
\hline & A. asparagoides (L.) Druce & $\mathrm{n}$ & $\mathrm{y}$ & native \\
\hline & A. buchananii Baker & $\mathrm{n}$ & $\mathrm{y}$ & native \\
\hline & A. migeodii Sebsebe & $\mathrm{n}$ & $\mathrm{y}$ & native \\
\hline & A. pendulus (Oberm.) Fellingham \& N.L. Mey. & $\mathrm{n}$ & $\mathrm{y}$ & native \\
\hline & A. psilurus Welw. ex Baker & $\mathrm{n}$ & $\mathrm{y}$ & native \\
\hline & A. suaveolens Burch. & $\mathrm{n}$ & $\mathrm{y}$ & unknown \\
\hline & A. virgatus Baker & $\mathrm{n}$ & $\mathrm{y}$ & native \\
\hline & A. africanus Lam. Var. africanus & $\mathrm{n}$ & $\mathrm{y}$ & native \\
\hline & A. africanus (Baker) Sebsebe var. puberulus & $\mathrm{n}$ & $\mathrm{y}$ & native \\
\hline & A. racemosus Willd. & $\mathrm{n}$ & $\mathrm{y}$ & unknown \\
\hline & A. saundersiae Baker & $\mathrm{n}$ & $\mathrm{y}$ & native \\
\hline & A. schroederi Engl. & $\mathrm{n}$ & $\mathrm{y}$ & native \\
\hline & A. setaceus (Kunth) Jessop & $\mathrm{n}$ & $\mathrm{y}$ & native \\
\hline \multirow[t]{6}{*}{ Bambara groundnut } & Vigna hosei (Craib) Backer var. pubescens & $\mathrm{y}$ & $\mathrm{y}$ & native \\
\hline & V. luteola (Jacq.) Benth. & $\mathrm{n}$ & $\mathrm{y}$ & native \\
\hline & V. oblongifolia A.Rich. & $\mathrm{n}$ & $\mathrm{y}$ & native \\
\hline & V. fischeri Harms & $\mathrm{n}$ & $\mathrm{y}$ & unknown \\
\hline & V. heterophylla A.Rich. subsp.ambacensis & $\mathrm{n}$ & $\mathrm{y}$ & native \\
\hline & V. racemosa (G.Don) Hutch. \& Dalziel & $\mathrm{n}$ & $\mathrm{y}$ & unknown \\
\hline
\end{tabular}


TABLE 2. Contd.

\begin{tabular}{|c|c|c|c|c|}
\hline Crop & Priority crop wild relatives for Malawi & $\begin{array}{l}\text { Included in } \\
\text { the SADC } \\
\text { inventory } \\
\text { (Yes/No) }\end{array}$ & $\begin{array}{l}\text { Related to } \\
\text { crop of global } \\
\text { importance } \\
\text { (Yes/No) }\end{array}$ & Status \\
\hline \multirow[t]{2}{*}{ Banana } & Ensete ventricosum (Welw.) Cheesman & $\mathrm{n}$ & $\mathrm{y}$ & native \\
\hline & Musa livingstonianum (J.Kirk) Cheesman & $\mathrm{n}$ & $\mathrm{y}$ & unknown \\
\hline \multirow[t]{4}{*}{ Bitter melon } & Momordica foetida Schumach.* & $\mathrm{y}$ & $\mathrm{n}$ & native \\
\hline & Coccinia adoensis (A. Rich.) Cogn.* & $\mathrm{n}$ & $\mathrm{n}$ & unknown \\
\hline & Momordica boivinii Baill. & $\mathrm{n}$ & $\mathrm{n}$ & native \\
\hline & M. friesiorum (Harms) C. Jeffrey & $\mathrm{n}$ & $\mathrm{n}$ & native \\
\hline \multirow{5}{*}{ Black pepper } & Peperomia exigua (Blume) Miq. & $\mathrm{n}$ & $\mathrm{n}$ & native \\
\hline & P. retusa (L.f.) A. Dietr. & $\mathrm{n}$ & $\mathrm{n}$ & native \\
\hline & Piper capense L. fil.subsp.capense ** & $\mathrm{n}$ & $\mathrm{n}$ & native \\
\hline & P. capense L. fil. var.brachyrhachis * & $\mathrm{n}$ & $\mathrm{n}$ & native \\
\hline & P. umbellatum L. & $\mathrm{n}$ & $\mathrm{n}$ & native \\
\hline Blue berry & Vaccinium exul Bolus ** & $\mathrm{n}$ & $\mathrm{n}$ & native \\
\hline \multirow[t]{5}{*}{ Cardamom } & Aframomum alboviolaceum (Ridl.) K. Schum. & $\mathrm{n}$ & $\mathrm{n}$ & native \\
\hline & A. albiflorum Lock & $\mathrm{n}$ & $\mathrm{n}$ & native \\
\hline & A. alboviolaceum (Ridl.) K. Schum. & $\mathrm{n}$ & $\mathrm{n}$ & native \\
\hline & A. angustifolium (Sonn.) K. Schum. & $\mathrm{n}$ & $\mathrm{n}$ & native \\
\hline & A. zambesiacum (Baker) K. Schum. subsp. Zambesiacum & $\mathrm{n}$ & $\mathrm{n}$ & unknown \\
\hline Cassava & Manihot glaziovii Müll. Arg.** & $\mathrm{n}$ & $\mathrm{y}$ & introduced \\
\hline Chinese/Indian mastard, Rape & Brassica juncea (L.) Czern. ** & $\mathrm{n}$ & $\mathrm{y}$ & introduced \\
\hline
\end{tabular}


TABLE 2. Contd.

\begin{tabular}{|c|c|c|c|c|}
\hline Crop & Priority crop wild relatives for Malawi & $\begin{array}{l}\text { Included in } \\
\text { the SADC } \\
\text { inventory } \\
\text { (Yes/No) }\end{array}$ & $\begin{array}{l}\text { Related to } \\
\text { crop of global } \\
\text { importance } \\
\text { (Yes/No) }\end{array}$ & Status \\
\hline \multirow[t]{6}{*}{ Clover } & Trifolium polystachyum Fresen. var. psoraleoides Welw. ex Hiern & $\mathrm{n}$ & $\mathrm{y}$ & native \\
\hline & T. pseudostriatum Baker f. & $\mathrm{n}$ & $\mathrm{y}$ & native \\
\hline & T. ruерpellianum Fresen. var. rueppellianum & $\mathrm{n}$ & $\mathrm{y}$ & native \\
\hline & T. semipilosum Fresen & $\mathrm{n}$ & $\mathrm{y}$ & native \\
\hline & T. simense Fresen. & $\mathrm{n}$ & $\mathrm{y}$ & native \\
\hline & T. usambarense Raub. & $\mathrm{n}$ & $\mathrm{y}$ & native \\
\hline \multirow[t]{8}{*}{ Coffee } & Coffea arabica $\mathrm{L}$. wild types $* *$ & $\mathrm{n}$ & $\mathrm{n}$ & native \\
\hline & C. eugenioides S.Moore * & $\mathrm{y}$ & $\mathrm{n}$ & unknown \\
\hline & C. ligustroides S.Moore* & $\mathrm{n}$ & $\mathrm{n}$ & unknown \\
\hline & C. mufindiensis Hutch. ex Bridson subsp. Mufindiensis* & $\mathrm{y}$ & $\mathrm{n}$ & unknown \\
\hline & C. mufindiensis Hutch ex Bridson subsp. australis * & $\mathrm{y}$ & $\mathrm{n}$ & native \\
\hline & C. mufindiensis Hutch ex Bridson subsp. lundaziensis * & $\mathrm{y}$ & $\mathrm{n}$ & native \\
\hline & C. mufindiensis Hutch ex Bridson subsp. pawekiana* & $\mathrm{y}$ & $\mathrm{n}$ & native \\
\hline & C. salvatrix Swynnerton \& Phillipson.* & $\mathrm{y}$ & $\mathrm{n}$ & native \\
\hline Cotton & Gossypium barbadense L.** & $\mathrm{y}$ & $\mathrm{n}$ & unknown \\
\hline \multirow[t]{7}{*}{ Cowpeas } & Vigna comosa Baker & $\mathrm{n}$ & $\mathrm{y}$ & native \\
\hline & V. phoenix Brummitt & $\mathrm{n}$ & $\mathrm{y}$ & native \\
\hline & V. scabra (L.f.)Sond subsp.scabra & $\mathrm{n}$ & $\mathrm{y}$ & unknown \\
\hline & V. schimperi Baker & $\mathrm{n}$ & $\mathrm{y}$ & native \\
\hline & V. unguiculata (E.Mey.) Marechal\& al. subsp.tenuis & $\mathrm{y}$ & $\mathrm{y}$ & native \\
\hline & V. adenantha (G.Mey.)Marechal \& al & $\mathrm{n}$ & $\mathrm{y}$ & unknown \\
\hline & V. antunesii Harms & $\mathrm{n}$ & $\mathrm{y}$ & native \\
\hline
\end{tabular}


TABLE 2. Contd.

\begin{tabular}{|c|c|c|c|c|}
\hline Crop & Priority crop wild relatives for Malawi & $\begin{array}{l}\text { Included in } \\
\text { the SADC } \\
\text { inventory } \\
\text { (Yes/No) }\end{array}$ & $\begin{array}{l}\text { Related to } \\
\text { crop of global } \\
\text { importance } \\
\text { (Yes/No) }\end{array}$ & Status \\
\hline & V. frutescens A.Rich. subsp.frutescens & $\mathrm{n}$ & $\mathrm{y}$ & native \\
\hline & V. gazensis Baker f. & $\mathrm{n}$ & $\mathrm{y}$ & native \\
\hline & V. nuda N.E.Br. & $\mathrm{n}$ & $\mathrm{y}$ & native \\
\hline & V. unguiculata (L.) Walp. subsp. unguiculata var. spontanea $* *$ & $\mathrm{n}$ & $\mathrm{y}$ & native \\
\hline & V. unguiculata (L.) Walp. subsp. pawekiae* & $\mathrm{y}$ & $\mathrm{y}$ & native \\
\hline & V. unguiculata (L.) Walp.subsp. pubescens* & $\mathrm{y}$ & $\mathrm{y}$ & unknown \\
\hline & V. unguiculata (L.) Walp.subsp.stenophylla ** & $\mathrm{y}$ & $\mathrm{y}$ & unknown \\
\hline & V. unguiculata (L.) Walp. subsp.tenuis * & $\mathrm{y}$ & $\mathrm{y}$ & native \\
\hline & V. unguiculata (Harms) Verdc. subsp. dekindtiana $* *$ & $\mathrm{y}$ & $\mathrm{y}$ & native \\
\hline & V. vexillata (L.) A.Rich. subsp.angustifolia & $\mathrm{n}$ & $\mathrm{y}$ & native \\
\hline & V. vexillata (L.) A. Rich. var.vexillata & $\mathrm{n}$ & $\mathrm{y}$ & native \\
\hline & V. kirkii (Baker) J.B.Gillett & $\mathrm{n}$ & $\mathrm{y}$ & native \\
\hline & V. platyloba Welw. ex Hiern & $\mathrm{n}$ & $\mathrm{y}$ & native \\
\hline & V.pygmaea R.E.Fr. & $\mathrm{n}$ & $\mathrm{y}$ & native \\
\hline & V. reticulata Hook.f. & $\mathrm{n}$ & $\mathrm{y}$ & native \\
\hline & V. schimperi Baker & $\mathrm{n}$ & $\mathrm{y}$ & native \\
\hline & V. juncea Milne-Redh. & $\mathrm{n}$ & $\mathrm{y}$ & native \\
\hline & V. nyangensis R.Mithen \& H.Kibblewhite & $\mathrm{n}$ & $\mathrm{y}$ & unknown \\
\hline & V. radicans Baker & $\mathrm{n}$ & $\mathrm{y}$ & native \\
\hline & V. frutescens subsp. frutescens A.Rich. var. buchneri (Harms) Verdc. & $\mathrm{n}$ & $\mathrm{y}$ & native \\
\hline & V. macrorhyncha (Harms) Milne-Redh. & $\mathrm{n}$ & $\mathrm{y}$ & native \\
\hline & V. oblongifolia A. Rich. var.parviflora (Baker) Verdc. & $\mathrm{n}$ & $\mathrm{y}$ & native \\
\hline
\end{tabular}


TABLE 2. Contd.

\begin{tabular}{|c|c|c|c|c|}
\hline Crop & Priority crop wild relatives for Malawi & $\begin{array}{l}\text { Included in } \\
\text { the SADC } \\
\text { inventory } \\
\text { (Yes/No) }\end{array}$ & $\begin{array}{l}\text { Related to } \\
\text { crop of global } \\
\text { importance } \\
\text { (Yes/No) }\end{array}$ & Status \\
\hline \multirow[t]{6}{*}{ Cucumber } & Coccinia mildbraedii Harms & $\mathrm{n}$ & $\mathrm{n}$ & native \\
\hline & Cucumis anguria L. anguria & $\mathrm{n}$ & $\mathrm{n}$ & native \\
\hline & C. hirsutus Sond & $\mathrm{n}$ & $\mathrm{n}$ & native \\
\hline & Oreosyce africana Hook. f. & $\mathrm{n}$ & $\mathrm{n}$ & unknown \\
\hline & Mukia maderaspatana (L.) M. Roem. & $\mathrm{n}$ & $\mathrm{n}$ & native \\
\hline & Oreosyce africana Hook. f. & $\mathrm{n}$ & $\mathrm{n}$ & unknown \\
\hline Date palm & Phoenix reclinata Jacq. ** & $\mathrm{y}$ & $\mathrm{n}$ & native \\
\hline Desmodium & Desmodium ospriostreblum Chiov. ** & $\mathrm{n}$ & $\mathrm{n}$ & introduced \\
\hline \multirow[t]{15}{*}{ Eggplant } & Solanum anguivi Lam. & $\mathrm{n}$ & $\mathrm{y}$ & native \\
\hline & S. tettense Klotzsch & $\mathrm{n}$ & $\mathrm{y}$ & native \\
\hline & S. aethiopicum $\mathrm{L}$. & $\mathrm{n}$ & $\mathrm{y}$ & native \\
\hline & S. dasyphyllum Schumach. & $\mathrm{n}$ & $\mathrm{y}$ & native \\
\hline & S. goetzei Dammer & $\mathrm{n}$ & $\mathrm{y}$ & native \\
\hline & S. incanum L. * & $\mathrm{n}$ & $\mathrm{y}$ & native \\
\hline & S. lichtensteinii Willd.* & $\mathrm{y}$ & $\mathrm{y}$ & native \\
\hline & S. richardii Dunal var.richardii & $\mathrm{n}$ & $\mathrm{y}$ & native \\
\hline & S. richardii Dunal var.burtt-davyi & $\mathrm{n}$ & $\mathrm{y}$ & native \\
\hline & S. torvum Sw. & $\mathrm{n}$ & $\mathrm{y}$ & native \\
\hline & S. aculeatissimum Jacq. & $\mathrm{n}$ & $\mathrm{y}$ & native \\
\hline & S. aculeatissimum Dunalvar.aculeatissimum & $\mathrm{n}$ & $\mathrm{y}$ & native \\
\hline & S. aureitomentosum Bitter $*$ & $\mathrm{y}$ & $\mathrm{y}$ & native \\
\hline & S. campylacanthum Hochst. ex A.Rich.* & $\mathrm{y}$ & $\mathrm{y}$ & native \\
\hline & S. chrysotrichum Schltdl. & $\mathrm{n}$ & $\mathrm{y}$ & introduced \\
\hline
\end{tabular}


TABLE 2. Contd

\begin{tabular}{|c|c|c|c|c|}
\hline Crop & Priority crop wild relatives for Malawi & $\begin{array}{l}\text { Included in } \\
\text { the SADC } \\
\text { inventory } \\
\text { (Yes/No) }\end{array}$ & $\begin{array}{l}\text { Related to } \\
\text { crop of global } \\
\text { importance } \\
\text { (Yes/No) }\end{array}$ & Status \\
\hline & S. macrocarpon L. & $\mathrm{n}$ & $\mathrm{y}$ & native \\
\hline & S. nigrum/retroflexum $\mathrm{L}$. & $\mathrm{n}$ & $\mathrm{y}$ & introduced \\
\hline & S. aculeastrum Dunal subsp. aculeastrum & $\mathrm{n}$ & $\mathrm{y}$ & native \\
\hline & S. delagoense Dunal & $\mathrm{n}$ & $\mathrm{y}$ & native \\
\hline & S. hispidum Pers. & $\mathrm{n}$ & $\mathrm{y}$ & native \\
\hline & S. schumannianum Dammer & $\mathrm{n}$ & $\mathrm{y}$ & native \\
\hline & S. seaforthianum Andrewsvar.disjunctum O.E.Schulz & $\mathrm{n}$ & $\mathrm{y}$ & native \\
\hline & S. terminale Forssk. subsp.terminale & $\mathrm{n}$ & $\mathrm{y}$ & native \\
\hline & S. mammosum L. & $\mathrm{n}$ & $\mathrm{y}$ & native \\
\hline & S. panduriforme E.Mey. & $\mathrm{n}$ & $\mathrm{y}$ & native \\
\hline & S. giganteum Jacq. & $\mathrm{n}$ & $\mathrm{y}$ & native \\
\hline & S. memphiticum J.F.Gmel. & $\mathrm{n}$ & $\mathrm{y}$ & native \\
\hline & S. pseudospinosum C.H.Wright & $\mathrm{n}$ & $\mathrm{y}$ & native \\
\hline & S.grossidentatum A. Rich. & $\mathrm{n}$ & $\mathrm{y}$ & native \\
\hline \multirow[t]{2}{*}{ Faba beans } & Vicia paucifolia Barker & $\mathrm{n}$ & $\mathrm{y}$ & unknown \\
\hline & V. paucifolia Bakersubsp. malosana (Baker) Verdc. * & $\mathrm{n}$ & $\mathrm{y}$ & native \\
\hline \multirow[t]{2}{*}{ Finger millet } & Eleusine indica $(\mathrm{L})$ Gaenth* & $\mathrm{y}$ & $\mathrm{y}$ & unknown \\
\hline & E. coracana (L.) Gaertn. subsp.africana** & $\mathrm{y}$ & $\mathrm{y}$ & unknown \\
\hline \multirow[t]{5}{*}{ Foxtail millet } & Setaria italica (L.) P.Beauv. ** & $\mathrm{n}$ & $\mathrm{n}$ & introduced \\
\hline & S. atrata Hackel $*$ & $\mathrm{n}$ & $\mathrm{n}$ & native \\
\hline & S. grandis Stapf & $\mathrm{n}$ & $\mathrm{n}$ & native \\
\hline & S. nigrirostris (Nees) Dur. \& Schinz & $\mathrm{n}$ & $\mathrm{n}$ & native \\
\hline & S. pumila (Poir.) Roem. \& Schult. & $\mathrm{n}$ & $\mathrm{n}$ & native \\
\hline
\end{tabular}


TABLE 2. Contd.

\begin{tabular}{|c|c|c|c|c|}
\hline Crop & Priority crop wild relatives for Malawi & $\begin{array}{l}\text { Included in } \\
\text { the SADC } \\
\text { inventory } \\
\text { (Yes/No) }\end{array}$ & $\begin{array}{l}\text { Related to } \\
\text { crop of global } \\
\text { importance } \\
\text { (Yes/No) }\end{array}$ & Status \\
\hline \multirow[t]{5}{*}{ Ginger } & Siphonochilus aethiopicus (Schweinf.) B.L.Burtt & $\mathrm{n}$ & $\mathrm{n}$ & native \\
\hline & S. parvus Lock & $\mathrm{n}$ & $\mathrm{n}$ & native \\
\hline & S. rhodesicus (T.C.E.Fr.) Lock & $\mathrm{n}$ & $\mathrm{n}$ & native \\
\hline & S. carsonii (Baker) Lock & $\mathrm{n}$ & $\mathrm{n}$ & native \\
\hline & S. kirkii (Hook.) B.L. Burtt & $\mathrm{n}$ & $\mathrm{n}$ & native \\
\hline Gourds & Lagenaria sphaerica (Sond.) Naudin & $\mathrm{n}$ & $\mathrm{n}$ & native \\
\hline \multirow[t]{6}{*}{ Grapes } & Vitis rotundifolia (Forssk.) Vahl** & $\mathrm{n}$ & $\mathrm{n}$ & native \\
\hline & V. cornifolia (Baker) Planch* & $\mathrm{n}$ & $\mathrm{n}$ & native \\
\hline & V. gracilis (Guill. \& Perr.) Suess. & $\mathrm{n}$ & $\mathrm{n}$ & native \\
\hline & V. integrifolia (Baker) Planch. & $\mathrm{n}$ & $\mathrm{n}$ & unknown \\
\hline & V. petiolata Hook. f. & $\mathrm{n}$ & $\mathrm{n}$ & unknown \\
\hline & V. quadrangularis $\mathrm{L}$. & $\mathrm{n}$ & $\mathrm{n}$ & native \\
\hline Hyacinth beans & Lablab purpureus (L.) Sweet subsp.uncinatus var.uncinatus* & $\mathrm{n}$ & $\mathrm{n}$ & native \\
\hline \multirow[t]{4}{*}{ Kaki/persimmon } & Diospyros abyssinica (Hiern) F. Whitesubsp.attenuata & $\mathrm{n}$ & $\mathrm{n}$ & native \\
\hline & D. loureiriana G. Don. subsp. loureiriana & $\mathrm{n}$ & $\mathrm{n}$ & native \\
\hline & D. quiloensis (Hiern) F. White & $\mathrm{n}$ & $\mathrm{n}$ & native \\
\hline & D. truncatifolia A.N. Caveney & $\mathrm{n}$ & $\mathrm{n}$ & native \\
\hline \multirow[t]{3}{*}{ Lettuce } & Lactuca attenuate Stebbins & $\mathrm{n}$ & $\mathrm{n}$ & native \\
\hline & L. glandulifera Hook. f. & $\mathrm{n}$ & $\mathrm{n}$ & native \\
\hline & L. paradoxa Sch. Bip. ex A. Rich. & $\mathrm{n}$ & $\mathrm{n}$ & native \\
\hline Lima bean & Macroptilium atropurpureum (Moç. \& Sessé ex DC.) Urb.* & $\mathrm{n}$ & $\mathrm{y}$ & unknown \\
\hline Livingstone potato & Plectranthus mandalensis Baker & $\mathrm{n}$ & $\mathrm{n}$ & native \\
\hline
\end{tabular}


TABLE 2. Contd

\begin{tabular}{|c|c|c|c|c|}
\hline Crop & Priority crop wild relatives for Malawi & $\begin{array}{l}\text { Included in } \\
\text { the SADC } \\
\text { inventory } \\
\text { (Yes/No) }\end{array}$ & $\begin{array}{l}\text { Related to } \\
\text { crop of global } \\
\text { importance } \\
\text { (Yes/No) }\end{array}$ & Status \\
\hline Lupine & Lupinus mexicanus Cerv.* & $\mathrm{n}$ & $\mathrm{y}$ & native \\
\hline \multirow[t]{7}{*}{ Millet } & Echinochloa haploclada (Stapf) Stapf $*$ & $\mathrm{n}$ & $\mathrm{n}$ & native \\
\hline & Echinochloa frumentacea Link $* *$ & $\mathrm{n}$ & $\mathrm{n}$ & introduced \\
\hline & E. jubata Stapf & $\mathrm{n}$ & $\mathrm{n}$ & native \\
\hline & E. pyramidalis (Lam.) Hitchc. \& Chase & $\mathrm{n}$ & $\mathrm{n}$ & native \\
\hline & E. colona $(\mathrm{L}$.$) Link { }^{* *}$ & $\mathrm{n}$ & $\mathrm{n}$ & native \\
\hline & E. crus-galli (L.) P.Beauv. & $\mathrm{n}$ & $\mathrm{n}$ & introduced \\
\hline & E. stagnina (Retz.) P.Beauv.(L).P. Beauv. & $\mathrm{n}$ & $\mathrm{n}$ & native \\
\hline \multirow[t]{4}{*}{ Olives } & Olea capensis $L$. & $\mathrm{n}$ & $\mathrm{n}$ & unknown \\
\hline & O. capensis L. subsp.macrocarpa & $\mathrm{n}$ & $\mathrm{n}$ & unknown \\
\hline & O. europaea L. subsp.cuspidata** & $\mathrm{y}$ & $\mathrm{n}$ & native \\
\hline & O. welwitschii (Knobl.) & $\mathrm{n}$ & $\mathrm{n}$ & native \\
\hline \multirow[t]{5}{*}{ Panicum } & Panicum adenophorum K.Schum. & $\mathrm{n}$ & $\mathrm{n}$ & native \\
\hline & P. nymphoides Renvoize* & $\mathrm{n}$ & $\mathrm{n}$ & native \\
\hline & P. lukwangulense Pilg. & $\mathrm{n}$ & $\mathrm{n}$ & native \\
\hline & P. miliaceum $\mathrm{L}$. & $\mathrm{n}$ & $\mathrm{n}$ & unknown \\
\hline & P. repens $\mathrm{L}$. & $\mathrm{y}$ & $\mathrm{n}$ & native \\
\hline \multirow[t]{7}{*}{ Pearl millet } & Cenchrus purpureus (Schumach.)Morrone ** & $\mathrm{y}$ & $\mathrm{y}$ & native \\
\hline & C. clandestinum Hochst. ex Chiov & $\mathrm{n}$ & $\mathrm{y}$ & introduced \\
\hline & C. geniculatus Thunb & $\mathrm{n}$ & $\mathrm{y}$ & native \\
\hline & C. polystachios L.subsp.polystachios & $\mathrm{n}$ & $\mathrm{y}$ & native \\
\hline & C. polystachios L.Morrone. subsp. atrichus & $\mathrm{n}$ & $\mathrm{y}$ & native \\
\hline & C. sphacelatum (Nees) T.Durand \& Schinz & $\mathrm{n}$ & $\mathrm{y}$ & native \\
\hline & C. ciliaris (L.) Link & $\mathrm{n}$ & $\mathrm{y}$ & unknown \\
\hline
\end{tabular}


TABLE 2. Contd

\begin{tabular}{|c|c|c|c|c|}
\hline Crop & Priority crop wild relatives for Malawi & $\begin{array}{l}\text { Included in } \\
\text { the SADC } \\
\text { inventory } \\
\text { (Yes/No) }\end{array}$ & $\begin{array}{l}\text { Related to } \\
\text { crop of global } \\
\text { importance } \\
\text { (Yes/No) }\end{array}$ & Status \\
\hline & C. atrichum Stapf \& C.E.Hubb & $\mathrm{n}$ & $\mathrm{y}$ & native \\
\hline & C. kirkii Stapf & $\mathrm{n}$ & $\mathrm{y}$ & native \\
\hline & C. macrourum Trin & $\mathrm{n}$ & $\mathrm{y}$ & native \\
\hline & C. mildraedii $\mathrm{Mez}$ & $\mathrm{n}$ & $\mathrm{y}$ & native \\
\hline & C. setosum (Sw.) Rich. & $\mathrm{n}$ & $\mathrm{y}$ & native \\
\hline & C. thunbergii Kunth & $\mathrm{n}$ & $\mathrm{y}$ & native \\
\hline & C. unisetus (Nees) Morrone & $\mathrm{n}$ & $\mathrm{y}$ & native \\
\hline Pigeon pea & Pearsonia cajanifolia (Baker) Polhill. subsp. cryptantha & $\mathrm{n}$ & $\mathrm{y}$ & native \\
\hline Plum & Prunus africana (Hook.f.) Kalkman & $\mathrm{n}$ & $\mathrm{n}$ & native \\
\hline \multirow[t]{3}{*}{ Potato } & Solanum tuberosum L. (wild types) & $\mathrm{y}$ & $\mathrm{y}$ & native \\
\hline & S. wendlandii Hook.f. & $\mathrm{n}$ & $\mathrm{y}$ & native \\
\hline & S. wrightii Benth. & $\mathrm{n}$ & $\mathrm{y}$ & native \\
\hline Pumpkin & Gunnera perpensa $\mathrm{L}$. & $\mathrm{n}$ & $\mathrm{n}$ & native \\
\hline \multirow[t]{2}{*}{ Quinoa } & Chenopodium procerum Hochst. ex Moq. & $\mathrm{n}$ & $\mathrm{n}$ & native \\
\hline & C. ambrosioides $\mathrm{L}$. & $\mathrm{n}$ & $\mathrm{n}$ & introduced \\
\hline \multirow[t]{5}{*}{ Raspberry } & Rubus iringanus Gust. & $\mathrm{n}$ & $\mathrm{n}$ & native \\
\hline & R. scheffleri Engl. & $\mathrm{n}$ & $\mathrm{n}$ & unknown \\
\hline & R. niveus Thunb* & $\mathrm{n}$ & $\mathrm{n}$ & introduced \\
\hline & R. ellipticus $\mathrm{Sm} . * *$ & $\mathrm{n}$ & $\mathrm{n}$ & introduced \\
\hline & R. rosifolius $\mathrm{Sm} . *$ & $\mathrm{n}$ & $\mathrm{n}$ & unknown \\
\hline
\end{tabular}


TABLE 2. Contd.

\begin{tabular}{|c|c|c|c|c|}
\hline Crop & Priority crop wild relatives for Malawi & $\begin{array}{l}\text { Included in } \\
\text { the SADC } \\
\text { inventory } \\
\text { (Yes/No) }\end{array}$ & $\begin{array}{l}\text { Related to } \\
\text { crop of global } \\
\text { importance } \\
\text { (Yes/No) }\end{array}$ & Status \\
\hline \multirow[t]{2}{*}{ Rhodes grass } & Chloris roxburghiana Schult. & $\mathrm{n}$ & $\mathrm{n}$ & unknown \\
\hline & Chloris pilosa Schumach. & $\mathrm{n}$ & $\mathrm{n}$ & introduced \\
\hline \multirow[t]{3}{*}{ Rice } & Oryza punctata Kotschy ex Steud.* & y & y & unknown \\
\hline & O. barthii A.Chev. ** & $\mathrm{y}$ & $\mathrm{y}$ & native \\
\hline & O. longistaminata A.Chev.\&Roehr. ** & $\mathrm{y}$ & $\mathrm{y}$ & native \\
\hline \multirow[t]{4}{*}{ Sesame } & Sesamum angolense Welw. & $\mathrm{n}$ & $\mathrm{n}$ & native \\
\hline & S. angustfolium (Oliver) Engl. & $\mathrm{y}$ & $\mathrm{n}$ & native \\
\hline & S. calycinum Welw. subsp.calycinum & $\mathrm{n}$ & $\mathrm{n}$ & unknown \\
\hline & S. calycinum Seidenst. ex H.-D.Ihlenfeldt subsp.pseudoangolense & $\mathrm{n}$ & $\mathrm{n}$ & unknown \\
\hline \multirow[t]{9}{*}{ Sorghum } & Sorghum almum (L) Parodi & $\mathrm{n}$ & $\mathrm{y}$ & native \\
\hline & S. bicolor (L.) Moench subsp. arundinaceum $* *$ & $\mathrm{y}$ & $\mathrm{y}$ & native \\
\hline & S. bicolor (L.) Moench subsp. bicolor ** & $\mathrm{n}$ & $\mathrm{y}$ & native \\
\hline & S. bicolor (L.) Moench subsp.drummondii** & $\mathrm{n}$ & $\mathrm{y}$ & native \\
\hline & S. bicolor (L.) Moench subsp.verticilliflorum** & $\mathrm{n}$ & $\mathrm{y}$ & native \\
\hline & S. halepense (L.) Pers.* & $\mathrm{n}$ & $\mathrm{y}$ & native \\
\hline & S. rigidifolium Stapf & $\mathrm{n}$ & $\mathrm{y}$ & native \\
\hline & S. sudanense (Piper) Stapf & $\mathrm{n}$ & $\mathrm{y}$ & native \\
\hline & S. versicolor Andersson & y & y & native \\
\hline \multirow[t]{4}{*}{ Soybean } & $\begin{array}{l}\text { Neonotonia wightii subsp.wightii (Wight \& Arn.) J.A. Lackey var } \\
\text {.longicauda (Schweinf.) J.A. Lackey }\end{array}$ & $\mathrm{n}$ & $\mathrm{n}$ & unknown \\
\hline & Ophrestia unifoliolata (Baker f.) Verdc. & $\mathrm{n}$ & $\mathrm{n}$ & native \\
\hline & Rhynchosia sublobata (Schumach. \& Thonn.) Meikle & $\mathrm{n}$ & $\mathrm{n}$ & native \\
\hline & S. spontaneum $L$. * & $\mathrm{n}$ & $\mathrm{n}$ & unknown \\
\hline
\end{tabular}


TABLE 2. Contd.

\begin{tabular}{|c|c|c|c|c|}
\hline Crop & Priority crop wild relatives for Malawi & $\begin{array}{l}\text { Included in } \\
\text { the SADC } \\
\text { inventory } \\
\text { (Yes/No) }\end{array}$ & $\begin{array}{l}\text { Related to } \\
\text { crop of global } \\
\text { importance } \\
\text { (Yes/No) }\end{array}$ & Status \\
\hline Spiny Cucumber & Cucumis metuliferus E.Mey. ex Naudin & $\mathrm{n}$ & $\mathrm{n}$ & native \\
\hline \multirow[t]{4}{*}{ Sugar cane } & Eriochrysis pallida Munro & $\mathrm{n}$ & $\mathrm{n}$ & native \\
\hline & Imperata cylindrica (L.) Raeusch. & $\mathrm{n}$ & $\mathrm{n}$ & unknown \\
\hline & Saccharum officinarum $\mathrm{L}^{* *}$ & $\mathrm{n}$ & $\mathrm{n}$ & native \\
\hline & S. spontaneum L.subsp.aegyptiacum ** & $\mathrm{y}$ & $\mathrm{n}$ & unknown \\
\hline \multirow[t]{18}{*}{ Sweet potato } & Ipomoea. coptica (L.) Roth ex Roem. \& Schult. var.acuta & $\mathrm{n}$ & $\mathrm{y}$ & native \\
\hline & I.turbinata Lag. & $\mathrm{n}$ & $\mathrm{y}$ & introduced \\
\hline & I. sinensis (Desr.) Choisy subsp. blepharosepala & $\mathrm{n}$ & $\mathrm{y}$ & native \\
\hline & I. blepharophylla Hallier f. & $\mathrm{n}$ & $\mathrm{y}$ & native \\
\hline & I. kituiensis Vatke & $\mathrm{n}$ & $\mathrm{y}$ & native \\
\hline & I. marginata (Desr.) Verdc. & $\mathrm{n}$ & $\mathrm{y}$ & native \\
\hline & I. mauritiana Jacq. & $\mathrm{n}$ & $\mathrm{y}$ & unknown \\
\hline & I. oenotherae (Vatke) Hallier f. & $\mathrm{n}$ & $\mathrm{y}$ & native \\
\hline & I. aquatica Forssk & $\mathrm{n}$ & $\mathrm{y}$ & native \\
\hline & I. barteri Baker var.barteri & $\mathrm{n}$ & $\mathrm{y}$ & native \\
\hline & I. cairica (L.) Sweet var. cairica & $\mathrm{n}$ & $\mathrm{y}$ & unknown \\
\hline & I. coptica (L.) Roth ex Roem. \& Schult. var. coptica & $\mathrm{n}$ & $\mathrm{y}$ & native \\
\hline & I. plebeia $\mathrm{R}$. Br. subsp.africana A. Meeuse & $\mathrm{n}$ & $\mathrm{y}$ & native \\
\hline & I. involucrata P.Beauv var.involucrata & $\mathrm{n}$ & $\mathrm{y}$ & native \\
\hline & I. muricata (L.) Jacq. & $\mathrm{n}$ & $\mathrm{y}$ & unknown \\
\hline & I. obscura (L.) Ker Gawl. var.sagittifolia Verdc. & $\mathrm{n}$ & $\mathrm{y}$ & native \\
\hline & I. obscura (L.) Ker Gawl. var.obscura & $\mathrm{n}$ & $\mathrm{y}$ & native \\
\hline & I. pes-tigridis $\mathrm{L}$. var. africana Hallier $\mathrm{f}$. & $\mathrm{n}$ & $\mathrm{y}$ & native \\
\hline
\end{tabular}


TABLE 2. Contd.

\begin{tabular}{|c|c|c|c|c|}
\hline Crop & Priority crop wild relatives for Malawi & $\begin{array}{l}\text { Included in } \\
\text { the SADC } \\
\text { inventory } \\
\text { (Yes/No) }\end{array}$ & $\begin{array}{l}\text { Related to } \\
\text { crop of global } \\
\text { importance } \\
\text { (Yes/No) }\end{array}$ & Status \\
\hline & I.-tigridis L. var .pes-tigridis & $\mathrm{n}$ & $\mathrm{y}$ & native \\
\hline & I. tenuirostris Steud. ex Choisy subsp.tenuirostris & $\mathrm{n}$ & $\mathrm{y}$ & native \\
\hline & I. crassipes Hook.var. crassipes & $\mathrm{n}$ & $\mathrm{y}$ & native \\
\hline & I. involucrata P. Beauv. var. operosa (C.H. Wright) Hallier f. & $\mathrm{n}$ & $\mathrm{y}$ & native \\
\hline & I. pileata Roxb. & $\mathrm{n}$ & $\mathrm{y}$ & native \\
\hline & I. dichroa Hochst. ex Choisy & $\mathrm{n}$ & $\mathrm{y}$ & native \\
\hline & $\begin{array}{l}\text { I. fulvicaulis (Hochst. ex Choisy)Boiss. ex Hallier f. var.asperifolia } \\
\text { (Hallier f.) Verdc. }\end{array}$ & $\mathrm{n}$ & $\mathrm{y}$ & native \\
\hline & I. hederifolia $\mathrm{L}$ & $\mathrm{n}$ & $\mathrm{y}$ & introduced \\
\hline & $\begin{array}{l}\text { I. fulvicaulis (Hochst. ex Choisy) Boiss. ex Hallierf. var.heterocalyx } \\
\text { (Schulze-Menz) Verdc. }\end{array}$ & $\mathrm{n}$ & $\mathrm{y}$ & native \\
\hline & I. linosepala Hallier f.subsp. alpina (Rendle) Lejoly \& Lisowski & $\mathrm{n}$ & $\mathrm{y}$ & native \\
\hline & I. rubens Choisy & $\mathrm{n}$ & $\mathrm{y}$ & native \\
\hline & I. pes-caprae (L.) R. Br. subsp. brasiliensis (L.) van Oststr. & $\mathrm{n}$ & $\mathrm{y}$ & native \\
\hline & I. eriocarpa $\mathrm{R} . \mathrm{Br}$. & $\mathrm{n}$ & $\mathrm{y}$ & native \\
\hline & I. trinervia Schulze-Menz & $\mathrm{n}$ & $\mathrm{y}$ & native \\
\hline & I. verbascoidea Choisy & $\mathrm{n}$ & $\mathrm{y}$ & native \\
\hline & I. welwitschii Vatke ex Hallier f. & $\mathrm{n}$ & $\mathrm{y}$ & native \\
\hline & I. wightii (Wall.) Choisy var.wightii & $\mathrm{y}$ & $\mathrm{y}$ & native \\
\hline Sword/Jack bean & Canavalia Africana Dunn* & $\mathrm{n}$ & $\mathrm{y}$ & unknown \\
\hline
\end{tabular}


TABLE 2. Contd.

\begin{tabular}{|c|c|c|c|c|}
\hline Crop & Priority crop wild relatives for Malawi & $\begin{array}{l}\text { Included in } \\
\text { the SADC } \\
\text { inventory } \\
\text { (Yes/No) }\end{array}$ & $\begin{array}{l}\text { Related to } \\
\text { crop of global } \\
\text { importance } \\
\text { (Yes/No) }\end{array}$ & Status \\
\hline \multirow[t]{6}{*}{ Teff } & Eragrostis tef (Zuccagni) Trotter $* *$ & $\mathrm{n}$ & $\mathrm{n}$ & native \\
\hline & E. aethiopica Chiov. & $\mathrm{n}$ & $\mathrm{n}$ & native \\
\hline & E. heterolomera StapF. ** & $\mathrm{n}$ & $\mathrm{n}$ & native \\
\hline & E. fastigiata Cope & $\mathrm{n}$ & $\mathrm{n}$ & native \\
\hline & E. sylviae Cope. & $\mathrm{n}$ & $\mathrm{n}$ & native \\
\hline & E. pilosa (L).P.Beauv. * & $\mathrm{n}$ & $\mathrm{n}$ & native \\
\hline Tobacco & Nicotiana rustica L. * & $\mathrm{n}$ & $\mathrm{n}$ & introduced \\
\hline Tomato & Solanum tarderemotum Bitter* & $\mathrm{n}$ & $\mathrm{n}$ & native \\
\hline \multirow[t]{5}{*}{ Yam bean } & Sphenostylis briartii (De Wild.) Baker f. & $\mathrm{n}$ & $\mathrm{n}$ & unknown \\
\hline & S. erecta (Baker f.) Hutch. ex Baker f. subsp.erecta & $\mathrm{n}$ & $\mathrm{n}$ & native \\
\hline & S. erecta (Baker f.) Hutch. ex Bakersubsp.obtusifolia (Harms) & $\mathrm{n}$ & $\mathrm{n}$ & unknown \\
\hline & Potter \& Doyle & & & \\
\hline & S. stenocarpa (Hochst. ex A. Rich.) Harms & $\mathrm{n}$ & $\mathrm{n}$ & unknown \\
\hline \multirow[t]{4}{*}{ Yam } & Dioscorea praehensilis Benth.** & $\mathrm{y}$ & $\mathrm{y}$ & native \\
\hline & D. hirtiflora Benth. subsp.orientalis* & $\mathrm{n}$ & $\mathrm{y}$ & native \\
\hline & D. asteriscus Burkill & $\mathrm{n}$ & $\mathrm{y}$ & native \\
\hline & D. bulbifera (L.) L** & $\mathrm{n}$ & $\mathrm{y}$ & native \\
\hline
\end{tabular}


TABLE 3. High priority taxa closely related to some cultivated crops and with potential use in crop improvement (*represents taxa with verified use in crop improvement)

\begin{tabular}{|c|c|c|c|c|}
\hline Related crop & ild relative taxa & Genepool & & \\
\hline Cassava & Manihot glaziovii Müll.Arg*. & GP2 & NA & NA \\
\hline Chinese/Indian mustard, Rape seed & Brassica juncea (L.) Czern. & GP1b & NA & NA \\
\hline Coffee Arabica & Coffea arabica (wild types) L.* & GP1b & $\mathrm{EN}$ & NA \\
\hline Coffee Arabica & C. eugenioides S. Moore & GP2 & $\mathrm{LC}$ & NA \\
\hline Coffee Arabica & C. ligustroides S. Moore & GP2 & VU & NA \\
\hline Coffee Arabica & C. mufindiensis Hutch. ex Bridson & GP2 & $\mathrm{LC}$ & NA \\
\hline Coffee Arabica & C. salvatrix Swynnerton \& Phillipson. & GP2 & $\mathrm{EN}$ & NA \\
\hline Coffee Arabica & C. mufindiensis Hutch ex Bridson subsp. australis Bridson & GP2 & NA & NA \\
\hline Coffee Arabica & C. mufindiensis Hutch ex Bridson subsp. lundaziensis Bridson & GP2 & NA & NA \\
\hline Coffee Arabica & C. mufindiensis Hutch ex Bridson subsp. pawekiana Bridson & GP2 & NA & NA \\
\hline Cotton & Gossypium barbadense L.* & GP1b & NA & NA \\
\hline Cowpeas & Vigna unguiculata (L.) Walp. var. spontanea (Schweinf.) Pasquet & GP1b & NA & NA \\
\hline Cowpeas & V. unguiculata (L.) Walp. subsp. pawekiae Pasquet & GP2 & NA & NA \\
\hline Cowpeas & V.unguiculata (L.) Walp. subsp. pubescens (R.Wilczek) Pasquet & GP2 & NA & NA \\
\hline Cowpeas & V. unguiculata (L.) Walp. subsp. stenophylla (Harv.) Marechal et al. & GP1b & NA & $\mathrm{LC}$ \\
\hline Cowpeas & V. unguiculata (L.) Walp. subsp. tenuis (E.Mey.) Marechal et al. & GP1b & NA & $\mathrm{LC}$ \\
\hline Cowpeas & V. unguiculata (L.) Walp. subsp. dekindtiana (Harms) Verdc. & GP1b & NA & $\mathrm{LC}$ \\
\hline Date palm & Phoenix reclinata Jacq. & GP1b & NA & $\mathrm{LC}$ \\
\hline Eggplant & Solanum incanum $\mathrm{L}$. & GP2 & NA & NA \\
\hline Eggplant & S. lichtensteinii Willd. & GP2 & NA & $\mathrm{LC}$ \\
\hline Eggplant & S. aureitomentosum Bitter & GP2 & NA & NA \\
\hline Eggplant & S. campylacanthum Hochst. exA. Rich. & GP2 & NA & $\mathrm{LC}$ \\
\hline Finger millet & Eleusine indica (L.) Gaertn & GP2 & NA & NA \\
\hline Finger millet & E. coracana (L.) Gaertn. subsp. africana (Keen.-O’Byrne) Hilu \& de Wet & GP1b & $\mathrm{LC}$ & $\mathrm{LC}$ \\
\hline Foxtail millet & Setaria italica (L.) P. Beauv. & GP1b & NA & NA \\
\hline
\end{tabular}




\begin{tabular}{|c|c|c|c|c|}
\hline Related crop & Crop wild relative taxa & $\begin{array}{l}\text { Genepool } \\
\text { concept }\end{array}$ & $\begin{array}{l}\text { IUNC } \\
\text { global } \\
\text { red } \\
\text { listing }\end{array}$ & $\begin{array}{l}2018 \\
\text { South Africa } \\
\text { plants red } \\
\text { listing }\end{array}$ \\
\hline Indian barnyard millet & E. frumentacea Link & GP1b & $\mathrm{LC}$ & NA \\
\hline Jack bean & Canavalia africana Dunn. & GP2 & NA & NA \\
\hline Millet/Indian Barnyard & Echinochloa colona (L.) Link & GP1b & $\mathrm{LC}$ & $\mathrm{LC}$ \\
\hline Millet/Japanese Barnyard & E. stagnina (Retz.) P.Beauv./ (L). P. Beauv. & GP1b & $\mathrm{LC}$ & $\mathrm{LC}$ \\
\hline Olives & Olea europaea L.subsp. cuspidata (Wall. ex G.Don) Cif. & GP1b & NA & NA \\
\hline Pearl millet & Pennisetum purpureus (Schumach.) Morrone* & GP2 & $\mathrm{LC}$ & NA \\
\hline Raspberry(black) & Rubus niveus Thunb & GP2 & NA & NA \\
\hline Raspberry(red) & R. ellipticus $\mathrm{Sm}$. & GP1b & NA & NA \\
\hline Raspberry(red) & R. rosifolius $\mathrm{Sm}$. & GP2 & NA & NA \\
\hline Rice & Oryza punctata Kotschy ex Steud.* & GP2 & $\mathrm{LC}$ & $\mathrm{LC}$ \\
\hline Rice & O. barthii A. Chev*. & GP1b & $\mathrm{LC}$ & NA \\
\hline Rice & O. longistaminata A. Chev. \& Roehr.* & GP1b & $\mathrm{LC}$ & $\mathrm{VU}$ \\
\hline Sorghum & Sorghum bicolor (L.) Moench subsp. arundinaceum (Desv.) de Wet and Harlan* & * GP1b & NA & $\mathrm{LC}$ \\
\hline Sorghum & S. bicolor (L.) Moench subsp. Drummondii (Steud.) de Wet and Harlan & GP1b & NA & LC \\
\hline Sorghum & S. bicolor (L.) Moench subsp. verticilliflorum (Steud.) de Wet and Harlan & GP1b & NA & NA \\
\hline Sorghum & S. halepense (L.) Pers. & GP2 & NA & NA \\
\hline Sorghum & S. bicolor (L.) Moench subsp. bicolor* & GP1b & NA & NA \\
\hline Sugar cane & Saccharum spontaneum L. subsp. aegyptiacum (Willd.) Hack* & GP1b & NA & NA \\
\hline Sugar cane & S. spontaneum L.* & GP2 & NA & NA \\
\hline Teff & Eragrostis tef (Zaccagni) trotter & GP2 & $\mathrm{LC}$ & NA \\
\hline Teff & E. heterolomera Stap F. & GP1b & NA & NA \\
\hline Teff & E. pilosa (L.) P. Beauv. & GP2 & NA & $\mathrm{LC}$ \\
\hline White guinea yam & Dioscorea praehensilis Beth. & GP1b & NA & $\mathrm{LC}$ \\
\hline
\end{tabular}

$\mathrm{NA}=$ taxa whose threat status is unknown (not assessed yet by the time of data collation), $\mathrm{LC}=$ Least concern, $\mathrm{VU}=\mathrm{vulnerable}, \mathrm{EN}=\mathrm{endangered}$ taxa; $\mathrm{GP} 1 \mathrm{~b}=$ taxa in the genepool as cultivated crops, GP2 = taxa in the secondary genepool 
(Hook.f.) Kalkman, and Oryza longistaminata A. Chev. \& Roehras Vulnerable (VU), and the remaining 103 taxa as Least Concern (LC).

In terms of potential use for crop improvement, 73 taxa have potential for crop improvement and 69 taxa were found to be in GP1b and GP2, and eleven taxa have verified use in crop improvement (Table 3). Based on these, $87 \mathrm{CWR}$ taxa were then categorised as of high priority; while 190 are low priority for conservation in a scenario where resources for conservation are limited.

\section{DISCUSSION}

The general checklist. The results of this study indicate, for the first time, existence of a relatively great diversity of CWR taxa (at species, subspecies and variety levels) in Malawi occurring across its regions. This provides an opportunity for establishing genetic reserves for in situ conservation of priority CWR across all agro-ecological zones of Malawi, capturing unique adaptive zones that possibly represent unique and/or rare genes useful for improvement of specific traits in crops. Collection and conservation of such taxa under ex situ, could provide a broad range of unique alleles specific for each agroecology. The general checklist had 446 taxa and $74.7 \%$ of which were native to Malawi, although they had the centre of diversity of their related crops elsewhere (Vincent et al., 2013).

It is important to note that more than $50 \%$ of these taxa had unknown threat status at both global and SADC regional levels. This was expected as most conservation institutions have different mandates, inadequate expertise in redlisting, as well as lacking adequate resources to do the redlisting exercise (Hunter and Heywood, 2011).The general checklist captured taxa of national, regional (SADC) and of global important crops, and of crops not cultivated in the country,but with wild relatives in Malawi.Related studies in Zambia, Mauritius and South Africa reported similar results of existence of CWR of regional and global priority (Ng'uni et al., 2017; Bissessur et al., 2019; Holness et al., 2019) an indication that the SADC region share the CWR diversity providing a cushioned platform for germplasm exchange. Zambia for example, took a step further by pooling such diversity to facilitate its utilisation in pre-breeding and crop improvement programmes (Ng'uni et al., 2017) and Malawi has similar plans.

Regarding the wild relatives of fodder and forage crops, it should be noted that only major fodder crops were considered, given their complex botanic classification, as noted by Vincent et al. (2013), and the inadequate information about the exact species regarded as crops in Malawi, as most fodder species occur in the wild. With such status, it is practically impossible to put under active conservation of such fodder unless well defined. Therefore, the total number of CWR occurring in Malawi could be slightly higher than 446, hence the checklist should be regarded as a working list and it should be updated whenever new information is available.

The prioritised checklist. Malawi's priority CWR inventory includes 277 taxa, of which $26.4 \%$ has potential for crop improvement, $33.6 \%$ were a priority to the SADC region (Allen et al.,2017; 2019), and 59.2\% taxa were related to crops of global importance (FAO, 2009; Vincent et al., 2013). With inter dependency on food and raw materials among nations (Khoury et al., 2010; Kell et al., 2015), and harmonised access to plant genetic resources at all levels (FAO, 2009; Dempewolf et al., 2014; Allen et al., 2019); presence of such taxa allows for continued germplasm exchange, and places Malawi in an important role as providing a pool of genetic diversity relevant to the improvement of crops that are important at global and regional levels.

In terms of conservation, this study provided fundamental information such as the amount of priority diversity for conservation 
and this will guide the formulation of specific conservation action plans for the priority taxa. However,to address the conservation needs of all priority taxa, the next step should be field mapping of such taxa to assess their current conservation status and have the real picture on the ground.

The criteria and methods used to prioritise CWR were tailored to the conservation of plant genetic resources context in Malawi. Due to differences in conservational needs, other countries and/or regions have used other criteria, or the same criteria but different prioritisation methods (Vincent et al., 2013; Allen et al., 2017; Allen et al., 2019); and this only shows that CWR prioritisation varies according to the different contexts.

About $12.6 \%$ of the priority taxa occurring in Malawi confirmed uses in crop improvement (Table 3 ) for traits such as pests and diseases resistance, drought tolerance and increase yield (Hajjar and Hodgkin, 2007). These were used to improve crops like cotton (Jafar et al., 2018), sorghum (Wilson et al., 2000; Jordan et al., 2004), rice (Khush et al., 2004; Brar, 2005), pearl millet (Hanna, 1989), cowpeas (Hajjar and Hodgkin, 2007) and sugarcane (Ramdoyal and Badaloo, 2002; Edmé et al., 2005). Availability of drought tolerance genes in taxa such as $O$. Barthii A. Chev. and $O$. Longistaminata A.Chev. \& Roehr. provides an opportunity for rice improvement whose cultivation in Malawi is confined to lake shore areas with reliable water sources.

The occurrence of taxa with genes controlling traits of economic importance has potential to improve agricultural productivity and diversified production considering that (i) the present food security in Malawi relies on a few crops such as maize, rice, cowpeas and a few minor crops whose genetic diversity has been significantly explored due to agricultural intensification and continuous selection for high yielding traits to meet high food demands; (ii) breeding for drought tolerance and pests and disease resistance is complex and resource demanding (Witcombe et al., 2007); and (iii) use of populations with known resistance and tolerance could potentially save on time, hence the need to take advantage of the available taxa with such genes to save on time and resources

About 73 of the priority taxa in the inventory have potential use for crop improvement, but only 3 taxa have ex situ collections at the national genebank; and these may need to be evaluated to benefit national breeding programme. However, it is important to note that information about taxa potential and confirmed use for crop improvement was not available for some taxa. With the use of modern breeding methods, distantly related taxa in the general checklist could potentially be useful in breeding programmes, and these taxa were not reflected in the inventory, implying that the number of priority taxa would increase with availability of such information.

The importance of the developed National Inventory cannot be over emphasised; its use has already been demonstrated through development of proposed national conservation strategy for CWR in Malawi, as part of the Darwin Initiative funded project "Bridging agriculture and environment: Southern African cropwildrelative regional network" that was initiated in April 2019. This immediate application shows its significance to conservation efforts in Malawi, and its availability will facilitate active and sustainable conservation of priority CWR, as noted by Maxted et al. (2015) and Magos Brehm et al. (2017). It could also facilitate utilisation of such taxa by breeders (Dempewolf et al., 2014; Zhang et al., 2017).

However, an inventory alone may not be sufficient for effective conservation of the identified taxa; other complementary analyses such as distribution and diversity analyses need to be considered in order to identify hot spots potential for active in situ conservation and designation of genetic reserves that capture broad range of diversity (Maxted, 2003). Such additional analyses can be useful in the identification of populations for in situ 
conservation that represent the genetic diversity in the wild (Maxted et al., 2012). More importantly, these analyses will assist in the identification of both in situ and ex situ conservation gaps of the priority CWR.

As formulation of a national CWR conservation strategy is in progress, the inventory can provide as background information such taxa distribution, native and threat status, taxon endemicity, rarity and potential use in crop improvement, to guide initial stages in conservation planning. As a temporary measure, we recommend that priority taxa with potential use for crop improvement, taxa that are endemic and threatened, should be priority for ex situ collections because they are vulnerable to localised natural and anthropogenic factors. We also note the insufficiency of information about the threat status of the priority taxa at national level, and with only few taxa considered for red listing at global level, relying on such information might be misleading in that taxa threatened at global level may not be threatened at national level. With such information gaps, it is recommended that threat assessments are conducted at national level to have a true reflection that adequately guide the formulation of strategic conservation actions of such taxa.

\section{CONCLUSION}

The development of the CWR inventory is a first step towards a comprehensive system that will systematically guide the conservation and sustainable utilisation of CWR in Malawi. The tool is timely, especially now when Malawi is facing challenges of loss of biodiversity and increased demand for food as the population continues to grow. However, more information on taxa ex situ and in situ conservation status is required to facilitate an effective conservation planning.

We recommend conducting ecogeographic surveys, diversity analyses, and modelling of climate change impact on their future distributions as next step towards effective conservation planning of CWR. The proposed studies will help verify status of CWR considering that there has been changes in land use in some sites where the species occurred. Threat assessment should also be considered for priority taxa as this ensures formulation of conservation actions that address the needs of threatened taxa. Although the inventory adequately covers taxa of important crops for Malawi, it should be updated whenever more information is available in order to make it relevant to the prevailing conservation needs.

\section{ACKNOWLEDGEMENT}

The authors are grateful to all national stakeholders from Forestry Research Institute of Malawi, Lilongwe University of Agriculture and Natural Resources;Department of Crop and Soil Sciences, Ministry of Agriculture; Departments of Agricultural Research Services and Crop Production, National Herbarium and Botanic Gardens of Malawi, Environmental Affairs Department and National Commission for Science and Technology for participating in species prioritisation and validation of the CWR inventory. We acknowledge the Government of Malawi, through Agricultural Productivity Programme for Southern Africa (APPSA) for financing this study. We also acknowledge Biodiversity Information Facility (GBIF) through Biodiversity Information for Development (BID) project code BIDAF2017-0265-SMA that supported wild sorghum data collation that was in this study.

\section{REFERENCES}

Allen, E., Gaisberger, H., Magos Brehm, J., Maxted, N., Thormann, I., Lupupa, T. and Kell, M. D. 2019. Acrop wild relative inventory for Southern Africa: A first step in linking conservation and use of valuable wild populations for enhancing food security. Plant Genetic Resources1-12. doi.10.1017/S1479262118000515. 
Allen, E., Kell, S., Magos Brehm, J. and Gaisberger, H. 2017. Priority CWR species of the SADC region. Harvard Dataverse, V1. doi:10.7910/DVN/HSXUVE

Bissessur, P., Baider, C., Boodia, N., Badaloo, G., Bégué, J., Jhumka, Z., Meunier, A., Mungroo, Y., Gopal, V., Kell, S.P., Magos Brehm, J., Thormann, I. and JaufeerallyFakim, Y. 2019. Crop wild relative diversity and conservation planning in two isolated oceanic islands of a biodiversity hotspot (Mauritius and Rodrigues). Plant Genetic Resources 17(2):174 184.

Brar, D. 2005. Broadening the genepool and exploiting heterosis in cultivated rice, In: Rice is life: Scientific perspectives for the 21st Century. In: Toriyama, K. Heong, K. and Hardy, B. (Eds.), Proceedings of the World Rice Research Conference, Tokyo and Tsukuba, Japan, 4-7 November 2004. Tokyo and Tsukuba.

CBD. 2000. Convention on biological diversity Secretariat: Global Strategy for Plant Conservation (GSPC). Retrieved 15 December 2016, from CBD: https:// www.cbd.int/gspc/objectives.shtm.

CBD. 2019. Convention on biological diversity communications. Retrieved 30 January 2019, from CBD: https://www.cbd.int/doc/ notifications/2015/ntf-2015-092-gspcen.pdf.

Dempewolf, H., Eastwood, R. J., Guarino, L., Khoury, C. K., Müller, J. V. and Toll, J. 2014. Adapting agriculture to climate change: A global initiative to collect, conserve, and use crop wild relatives. Agro ecology and Sustainable Food Systems 38(4):369-377. doi:10.1080/21683565. 2013.870629.

Dombo, A., Da Costa, E. and Neto, G. 2012. Malawi Plant Red List data 2002. (J.S. Goldinged). Retrieved 23 July 2018, from https://www.nationalredlist.org/malawiplant-red-data-list-2002/..

Edme', S. J., Miller, J. D., Glaz, B. and Comstock, P. Y. 2005. Genetic contribution to yield gains in the Florida sugarcane industry across 33 years. Crop Science Journal 45:92-97. Retrieved from https:// naldc.nal.usda.gov/download/3378/PDF

FAO. 1996. The state of Malawi 's Plant Genetic Resources: Country Report by Malawi Governement submiited to FAO. Rome, Italy: FAO. Retrieved from http:// www.fao.org

FAO. 2009. Food and Agriculture Organization of the United Nations. Retrieved 2017, from http://www.planttreaty.org/

FAO. 2012. Food and Agricultural Organization of the United Nations: Synthetic account of the second global plan of action for plant genetic resources for food and agriculture. Commission on Plant Genetic Resources for Food and Agriculture. Retrieved 12 October, 2017, from http://www.fao.org/ docrep

FAO. 2019. Food and Agriculture Organization of the United Nations-FAOSTAT data for Malawi-crops and livestock products export value-Malawi. Retrieved 02 September 2019, from http://www.fao.org/ faostat/en/\#data/TP

FAO. (n.d.). Food and Agriculture Organization of the United Nations: Definition and classification of commodities. Rome. Retrieved 2019, from http://www.fao.org/waicent/faoinfo/ economic/faodef/faodefe.htm

FAOSTAT. 2016. Food and Agriculture Organization of the United Nations. Retrieved 2016, from http://faostat.fao.org/ default.aspx

GBIF. 2017. Global biodiversity information facility. Plant occurrence data sets for Malawi. Retrieved 14 March 2017,from https://www.gbif.org/occurrence/search ?q=plant $\% 20$ occurrence $\% 20$ data $\% 20$ sets \& country=MW

Hajjar, R. and Hodgkin, T. 2007. The use of wild relatives in crop improvement: A survey of developments over the last 20 years. Euphytica 156:1-13.doi:10.1007/ s10681-007-9363-0. 
Hanna, W. 1989. Characteristics and stability of a new cytoplasmic-nuclear male sterile source in pearl millet. Crop Science 29: 1457-1459. doi:10.2135/cropsci 1989. 0011183X002900060026x.

Harlan, J. and de Wet, J. 1971. Towards a rational classification of cultivated plants. Taxon 20:509- 517.doi: 10.2307/1218252.

Holness, S., Hamer, M., Magos Brehm, J. and Raimondo, D. 2019. Priority areas for the in situ conservation of crop wild relatives in South Africa. Plant Genetic Resources 17(2):115-127.

Hyde, M., Wursten, B., Ballings, P. and Coates Palgrave, M. 2018. Flora of Malawi: Species data: List of families. Retrieved 10 November 2018, from www.malawiflora. com/speciesdata/index.php.

Hunter, D. and Heywood, V. 2011. Crop wild relatives. Amanual of In situ Conservation. In: Hunter, D. and Heywood, (Eds.), Washington DC, USA: Bioversity international: Earthscan. pp. 211-295.

Ishimaru, T., Hirabayashi, H., Ida, M., Takai, T., San-Oh, Y. A. and Yoshinaga, S. 2010. A genetic resource for early-morning flowering trait of wild rice Oryza officinalis to mitigate high temperature-induced spikelet sterility at anthesis. Annals of Botany 106:515-520.

IUCN. 2018. The International Union of Nature Conservation Red List of Threatened Species. Retrieved 30 November 2018, from http://www.iucnredlist.org

Jafar, M., Ramesh, B., Satish, K. G., Kelly, P., Ibrokhim, Y. A. and Kumpatla, S. P. 2018. Wild relatives of maize, rice, cotton, and soybean: Treasure troves for tolerance to biotic and abiotic stresses. Front Plant Science 9(22). doi: 10.3389/fpls.2018. 00886.

Jordan, J., Butler, D., Henzell, B., Drenth, J. and McIntyre, L. 2004. Diversification of Australian sorghum using wild relatives, New directions for a diverse planet. Proceedings of the 4th International Crop Science Congress, Brisbane, Australia,26
Sep-1 Oct2004. Brisbane,: The regional institute online publishing.

Kell, S., Qin, H., Chen, B., Ford-Lloyd, B., Wei, W., Kang, D. and Maxted, N. 2015. China's crop wild relatives: diversity for agriculture. Agriculture Ecosystem and Environment 209:138-154. doi.10.1016/ j.agee.2015.02.012 0167-8809/ã 2015.

Khoury, C., Laliberte', B. and Guarino, L. 2010. Trends in ex situ conservation of plant genetic resources: a review of global crop and regional conservation strategies. Genetic Resources and Crop Evolution 57:625-639. doi.org/10.1007/s10722-0109534-z.

Khush, G., Angeles, E., Virak, P. S. and Brar, D. 2004. Breeding rice for resistance to Tungro virus at IRRI. SABRAO Journal of Breeding and Genetics 2(36):101-106. Retrieved from http://eprints.icrisat.ac.in/ 2899/

Magos Brehm, J., Saifan, S., Taifour, H., Abu Laila, K., Al-Assaf, A., Al-Oqlah, A., AlSheyab, F., Bani-Hani, R., Ghazanfar, S., Haddad, N., Shibli, R., Abu Taleb, T., bint Ali, B. and Maxted, N. 2016. Crop wild relatives, a priority in Jordan? - Developing a national strategy for the conservation of plant diversity in Jordan using a participatory approach." In: Maxted, N., Dulloo, M.E. and Ford-Lloyd, B.V. (Eds.). Enhancing Crop Genepool Use: Capturing Wild Relative and Landrace Diversity for Crop Improvement. CAB International, Wallingford, UK. pp. 172-188.

Magos Brehm, J., Kell, S., Thormann, I., Gaisberger, H., Dulloo, M. and Maxted, N. 2017. Interactive toolkit for crop wild relative conservation planning version. Accessed on htt://www.cropwildrelatives. org/conservation-toolkit/

Maxted, N. 2003. Conserving the genetic resources of crop wild relatives in European protected areas. Emerging tools and strategies. Biological Conservation 113(3):411-417.doi.10.1016/S00063207(03)00123-X. 
Maxted, N., Ford-Lloyd, B. V., Jury, S., Kell, S. and Scholten, M. 2006. Towards a definition of a crop wild relative. Biodiversity and Conservation. Conservational Biology 15:2673-2685. doi 10.1007/s 10531-005-5409-6.

Maxted, N. and Kell, S. 2009. Establishment of a global network for the In situ conservation of crop wild relatives: Status and needs. Thematic Background Study. Retrieved 20 December 2016, from http:// www.fao.org/docrep

Maxted, N., Kell, S., Ford-Lloyd, B., Dulloo, E. and Toledo, Á. 2012. Toward the systematic conservation of global crop wild relative diversity. Crop Science 52(2):774785. doi:10.2135/cropsci2011.08.0415.

Maxted, N., Avagyan, A., Frese, L., Iriondo, J., Kell, S.P., MagosBrehm, J., Singer, A. and Dulloo, E. 2015. Conservation planning for crop wild relative diversity. pp. 88-108. In: Redden R., Yadav S.S, Maxted N., Dulloo M.E., Guarino L. and Smith P. Hoboken (Eds.). Crop Wild Relatives and Climate Change. John Wiley \& Sons Inc, NJ, USA.

Ng'uni, D.,Munkombwe, G., Mwila, G., Dulloo, E., Thormann, I., Gaisberger,H., Maxted, N., Magos Brehm, J. and Kell, S. 2017.Technical Background Document to the National Strategic Action Plan for the Conservation and Sustainable Use of Cop Wild Relatives in Zambia. https://doi.org/ 10.7910/DVN/VYZZFF, Harvard Dataverse, V1.

Plaza, M., Andujar, I, Vilanova, S., Gramazio, P., Herraiz, F.J. and Prohens, J. 2014. Conventional and phenomics characterization provides insight into the diversity and relationships of hypervariable scarlet (Solanum aethiopicum L.) and gboma (S. macrocarpon L.) eggplant complexes. Frontier Plant Science. doi.org/10.3389/fpls.2014.00318.

Raimondo, D., von Staden, L., Foden, W., Victor, J., Helme, N., Turner, R. and
Kamundi, D. 2009. Red List of South African Plants. Retrieved from South African National Biodiversity Institute : http://redlist.sanbi.org/genera.php? generalike $=p$

Ramdoyal, K. and Badaloo, H. 2002. Prebreeding in sugarcane with an emphasis on the Programme of the Mauritius Sugar Industry Research Institute. Managing Plant Genetic Diversity. https://www. researchgate.net/publication/267260870. Accessed on 2 October 2019.

The Plant List. 2013. Retrieved November 2018, from http://www.theplantlist.org/

The Royal Botanical Gardens-KEW. 2017. KEW herbarium -specimens. Richmond, London, United Kingdom. Retrieved 2017, from http://specimens.kew.org/herbarium UN. 2015. United Nations: Sustainable Development goals 2015-2030. Retrieved 6 November 2018, fromhttps://www.un. org/sustainabledevelopment/sustainabledevelopment-goals/.

USD, Agricultural Research Service, National Plant Germplasm System. 2018. Germplasm Resources Information Network (GRIN-Taxonomy). National Germplasm Resources Laboratory, Beltsville, Maryland. Retrieved November 2018, from https://npgsweb.ars-grin.gov/ gringlobal/taxonomydetail.

Vincent, H., Wiersema, J., Kell, S., Fielder, H., Dobbie, S., Castañeda-Álvarez, N. P. and Maxted, N. 2013. A prioritized crop wild relative inventory to help underpin global food security. Conservation 167:265-275. doi.org/10.1016/j.biocon.2013.08.011.

Wiersema, J. and León, B. 2016.The GRIN Taxonomy Crop Wild Relative Inventory. In: N. Maxted, N., Dulloo, M.E. and Bryan Ford-Lloyd (Eds.). Enhancing Crop Genepool Use. Capturing Wild Relative and Landrace Diversity for Crop Improvement. $\mathrm{CAB}$ International, Wallingford, UK. 42:453-457. doi :10.1079/978178064 6138.0000 . 
Williamson, J. 2005. Useful plants of Malawi. Witcombe, J., Hollington, P., Howarth, C., (Third Edition) Montfort Press, Limbe, Reader, S. and Steele, K. 2007. Breeding Malawi.

Wilson, J., Hess, D. and Hanna, W. 2000. Resistance to Striga hermonthica in wild accessions of the primary gene pool of Pennisetum glaucum. Phytopathology 90: 1169-1172. Retrieved 7 July 2019, from https://naldc.nal.usda.gov/download/ 33951/PDF for abiotic stresses for sustainable agriculture. Royal Society. doi:10.1098/ rstb.2007.2179.

Zhang, H., Mittal, N., Leamy, L.J., Barazani, O. and Song, B.H. 2017. Back into the wild - Apply untapped genetic diversity of wild relatives for crop improvement. Evolutionary Applications 10:5-24. doi: 10.1111/eva.12434. 